\title{
Structure of the TRPV1 ion channel determined by electron cryo-microscopy
}

\author{
Maofu Liao ${ }^{1 *}$, Erhu $\mathrm{CaO}^{2 *}$, David Julius ${ }^{2} \&$ Yifan Cheng
}

Transient receptor potential (TRP) channels are sensors for a wide range of cellular and environmental signals, but elucidating how these channels respond to physical and chemical stimuli has been hampered by a lack of detailed structural information. Here we exploit advances in electron cryo-microscopy to determine the structure of a mammalian TRP channel, TRPV1, at 3.4 A resolution, breaking the side-chain resolution barrier for membrane proteins without crystallization. Like voltage-gated channels, TRPV1 exhibits four-fold symmetry around a central ion pathway formed by transmembrane segments 5-6 (S5-S6) and the intervening pore loop, which is flanked by S1-S4 voltage-sensor-like domains. TRPV1 has a wide extracellular 'mouth' with a short selectivity filter. The conserved 'TRP domain' interacts with the S4-S5 linker, consistent with its contribution to allosteric modulation. Subunit organization is facilitated by interactions among cytoplasmic domains, including amino-terminal ankyrin repeats. These observations provide a structural blueprint for understanding unique aspects of TRP channel function.

TRP channels represent a large and diverse family of non-selective cation channels that respond to a wide range of chemical and physical stimuli $^{1,2}$. Genetic and pharmacological studies highlight the importance of TRP channels in numerous biological processes ranging from calcium adsorption to sensory transduction ${ }^{3}$, and thus elucidating how these channels respond to physiological stimuli or drugs is relevant to understanding disorders affecting every major organ system in the body. TRP channels are believed to resemble voltage-gated potassium $\left(\mathrm{K}_{\mathrm{V}}\right)$ or sodium $\left(\mathrm{Na}_{\mathrm{V}}\right)$ channels in overall transmembrane topology and subunit organization ${ }^{1}$. However, aside from the fact that some TRP channels exhibit mild voltage sensitivity ${ }^{4}$, they otherwise share little in common with voltage-gated ion channels (VGICs) with regards to pharmacological and biophysical properties. Whereas crystal structures have been determined for VGICs ${ }^{5-8}$, this has not been accomplished for any member of the TRP channel family. Thus, it is currently unknown whether, or to what extent, TRP and $\mathrm{K}_{\mathrm{V}}$ or $\mathrm{Na}_{\mathrm{V}}$ channels share a common structural core, or how structural similarities and differences account for unique functionalities.

TRPV1 is the receptor for capsaicin, the pungent agent from chili peppers that elicits burning pain ${ }^{9}$. It is also the founding member of a subfamily of thermosensitive TRP channels that enable somatosensory neurons, or other cell types, to detect changes in ambient temperature. TRPV1 is activated by noxious heat and modulated by inflammatory agents, such as extracellular protons and bioactive lipids, which contribute to pain hypersensitivity ${ }^{9-13}$. TRPV1 is arguably the best-characterized member of the vertebrate TRP family; its widely validated role in pain physiology and the availability of well-characterized pharmacological agents make it a 'poster child' for elucidating basic principles underlying TRP channel function and structure. Moreover, TRPV1 and other somatosensory TRP channels are considered important targets for analgesic drugs ${ }^{12,13}$, providing further impetus to determine a structure for any member of this extended protein family.

In recent years, single-particle electron cryomicroscopy (cryo-EM) has enabled three-dimensional (3D) reconstruction of large protein complexes to near-atomic resolution ${ }^{14-17}$, but analysis of small membrane proteins, such as TRP channels ${ }^{18-20}$, remained at low resolution. Here, we exploit a newly developed direct electron detector and new imageprocessing algorithms to correct motion-induced image blurring and improve signal and contrast of single-particle cryo-EM images ${ }^{14,15}$. With these tools, we determine the structure of TRPV1 at $3.4 \mathrm{~A}$ resolution without crystallization. Thus, in addition to revealing a TRP channel structure, we showcase single-particle cryo-EM as a powerful and transformative advance in the structural analysis of membrane proteins.

\section{General architecture of TRPV1}

We first identified a rat TRPV1 deletion mutant with enhanced biochemical stability (Extended Data Figs 1 and 2). When expressed in mammalian cells or oocytes this modified subunit responded to numerous TRPV1 stimuli, including capsaicin, resiniferatoxin, extracellular protons, spider toxins, and heat, and like the wild-type channel showed relatively high permeability for calcium ${ }^{21,22}$. Thus, biophysical and structural information gleaned from this minimal TRPV1 construct should accurately reflect properties of the wild-type channel.

We initiated structural analysis using negative-stain EM (Extended Data Fig. 3) followed by cryo-EM 3D reconstruction of images recorded at $200 \mathrm{kV}$ with a phosphor scintillator-based complementary metaloxide-semiconductor (CMOS) camera (Extended Data Figs 4 and 5). We then collected a cryo-EM data set at $300 \mathrm{kV}$ using a direct detection camera following newly implemented procedures of dose fractionation and motion correction ${ }^{15}$. Thon rings were visible to $\sim 3 \AA$ in the Fourier power spectrum of almost every motion-corrected image (Fig. 1a, b and Extended Data Fig. 6). Two-dimensional (2D) class averages of particle images (Fig. 1c and Extended Data Fig. 7) showed greater detail than those calculated from CMOS camera images (Extended Data Fig. 4e, f), demonstrating considerable improvement of data quality. The final 3D reconstruction of TRPV1 has an overall resolution of $3.4 \AA$, using gold-standard Fourier shell correlation $(F S C)=0.143$ criteria (Fig. 1d-g and Extended Data Fig. 8) ${ }^{23}$.

Side-chain densities of most residues within transmembrane helices S1-S6 were clearly resolved, as well as the TRP domain following 

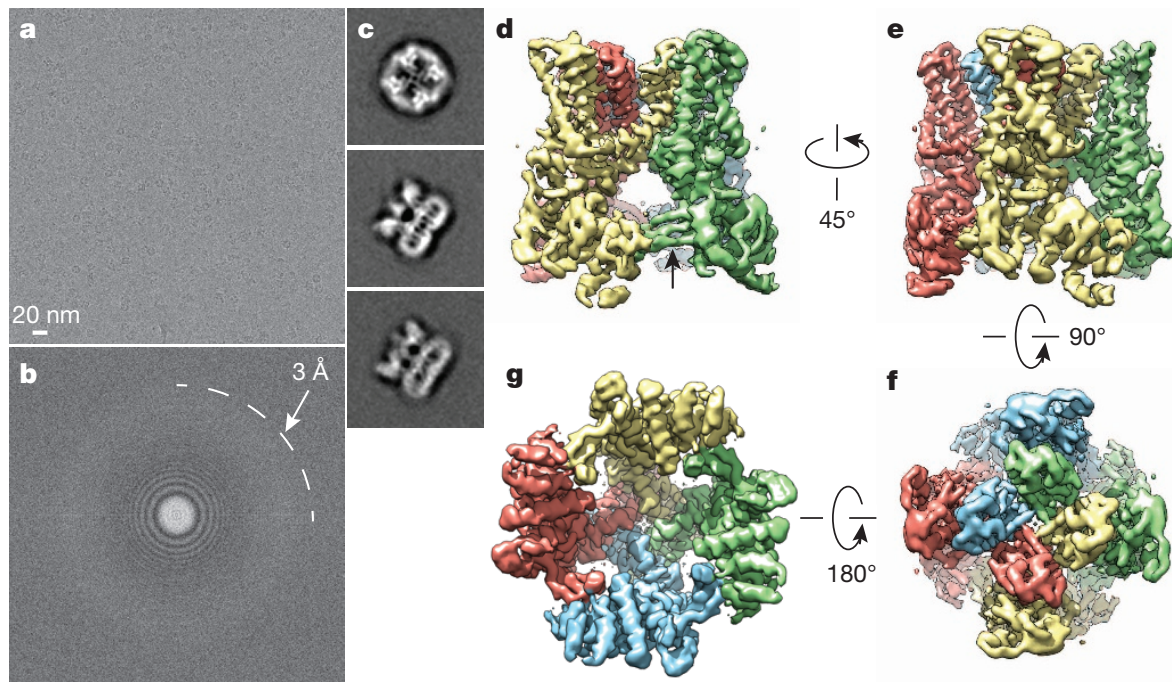

f

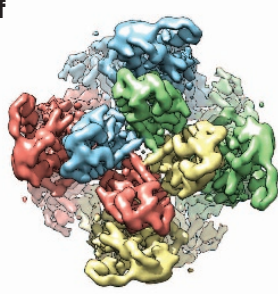

Figure $1 \mid 3 D$ reconstruction of TRPV1 determined by single-particle cryo-EM. a, Representative electron micrograph of TRPV1 protein embedded in a thin layer of vitreous ice recorded at a defocus of $1.7 \mu \mathrm{m}$. b, Fourier transform of micrograph shown in a, with Thon rings extending to nearly $3 \AA$. c, Enlarged views of three representative 2D class averages show fine features of

S6 and most $(\sim 80 \%)$ residues in the $\mathrm{N}$-terminal segment connecting the ankyrin repeat domain (ARD) to S1 (Extended Data Fig. 9a-d). In other cytoplasmic regions, densities were less well resolved, but antiparallel $\beta$-strands were well separated, indicating that cytoplasmic domains were resolved to $\leq 4.8 \AA$ (Fig. 1d, arrow). Densities for the first two ankyrin repeats were absent in our 3D density map (Fig. 1 and Extended Data Figs 5 and 8), even though the entire ARD was present in the expressed protein. Nonetheless, the crystal structure of isolated TRPV1 ankyrin repeats ${ }^{24}$ could be nicely docked to the density map as a rigid body without modification, as confirmed by bulkyside-chain densities (Extended Data Fig. 9e). Finally, we constructed an atomic model of TRPV1 in which residues L360 to A719 were built de novo, and L111 to E359 were docked from the ankyrin repeat crystal structure (Fig. 2). In the carboxy-terminal domain, we could trace the main chain, but side-chain densities were not sufficient for de novo model building.

Our TRPV1 structure demonstrates that, despite the rather low $(<20 \%)$ sequence similarity between TRPs and VGICs, these two channel families share a similar tetrameric architecture in which subunits are arranged in four-fold symmetry around a central ion permeation path. Each subunit consists of six transmembrane $\alpha$-helices (S1-S6) that span the lipid bilayer (Fig. 2 and Extended Data Fig. 8), plus a re-entrant loop with a pore helix located between S5 and S6 that together assume an 'inverted teepee' arrangement resembling that of VGICs (Fig. 2c).

Domains unique to TRP channels are immediately evident. First, we see four of six known ankyrin repeats at the $\mathrm{N}$ terminus, arranged as described previously ${ }^{24}$, followed by a linker (P360-V415) that is conserved among TRPV subtypes and which connects the ARD to the preS1 helix (Fig. 3). This linker forms a tightly packed domain containing $\beta$-strand and $\alpha$-helical elements, and is sandwiched between the sixth ankyrin repeat on one side and the pre-S1 helix and TRP domain on the other. Interestingly, an antiparallel $\beta$-sheet from the linker region and a $\beta$-strand from the $C$ terminus make contact with two ankyrin repeats from an adjacent subunit (Figs $1 d, 2 a$ and $3 b$ ), an interaction that probably has a role in channel assembly, reminiscent of the T1 domain in $\mathrm{K}_{\mathrm{V}}$ channels. Another unique feature is the extended and kinked interfacial helix following S6, which corresponds to the signature 'TRP domain' found in many TRP channels ${ }^{2}$. This domain is strategically located to interact with both the S4-S5 linker and pre-S1 helix (E416-R428), probably providing a physical substrate through which stimuli allosterically affect pore conformation (Fig. 3). tetrameric channel complex. $\mathbf{d}-\mathbf{g}$, 3D density map of TRPV1 channel filtered to a resolution of $3.4 \AA$ (scaled to atomic structure) with each subunit colour-coded. Four different views of the channel are shown, from side $(\mathbf{d}, \mathbf{e})$, top (f) and bottom ( $(\mathbf{g})$. The arrow in panel $\mathbf{d}$ indicates $\beta$-sheet structure in the cytosolic domain of TRPV1.

\section{The transmembrane core}

In VGICs, the transmembrane core is organized into two distinct clusters that include the central ion-conducting pore formed by tetrameric assembly of S5-P-S6 domains, and surrounding S1-S4 voltagesensing domains ${ }^{25,26}$. Each S1-S4 domain associates with the S5-P-S6 region of an adjacent subunit, forming a pinwheel-like structure through this 'domain swap' organization. Notably, we find that TRPV1 adopts this same overall configuration (Fig. 2d), despite minimal sequence and functional similarity to $\mathrm{VGICs}^{1,4}$. When pore regions (S5-P-S6) of three channels (TRPV1, $\mathrm{K}_{\mathrm{V}} 1.2-2.1$ chimaera and $\mathrm{Na}_{\mathrm{V}} \mathrm{Ab}$ ) are aligned, the relative positions of $\mathrm{S} 1-\mathrm{S} 4$ domains differ, even though the domains themselves show substantial overlap (Fig. 4a, b), perhaps reflecting intrinsic structural differences or distinct physiological states (for example, open, closed or desensitized). Within each TRPV1 subunit, the S1-S4 and S5-P-S6 units are connected by a helical S4-S5 linker that runs parallel to the membrane, again reminiscent of VGICs (Figs 3 and $4 \mathrm{c}$ and Extended Data Fig. 10a).

In VGICs, S3 and S4 helices engage in extensive side-chain interactions, forming a charge-transfer centre that facilitates movement of $\mathrm{S} 4$ in response to changes in membrane potential ${ }^{5,7}$. Interestingly, the equivalent region of TRPV1 lacks charged side chains and is, instead, replete with aromatic residues (Fig. $4 \mathrm{~d}$ and Extended Data Fig. 10b). The importance of this aromatic cluster is underscored by studies demonstrating that replacement of these amino acids (Y441, Y444, Y554 and Y555) with small non-aromatic residues produces non-functional channels ${ }^{27,28}$. Such hydrophobic packing might impart rigidity to the S1-S4 domain, allowing it to serve as a relatively stationary anchor upon which the S4-S5 linker moves to facilitate TRPV1 gating. Indeed, as discussed in the accompanying study ${ }^{29}$, the entire S1-S4 domain remains static during channel activation, but provides an external surface for the binding of lipophilic ligands, such as capsaicin and resiniferatoxin. Thus, unique biochemical interactions within this structurally conserved S1-S4 domain probably reflect differential sensitivities of VGICs and TRPs to specific physiologic stimuli (that is, voltage versus lipid metabolites).

\section{The TRP domain and S4-S5 linker}

The TRP domain, a 23-25-amino-acid-long region located just after S6, is found in many TRP family members and has been proposed to engage in subunit assembly or allosteric modulation of channel gating ${ }^{1,2,30}$. We find that the TRP domain assumes a $\alpha$-helical structure that runs parallel to the inner leaflet of the membrane by virtue of a sharp bend 

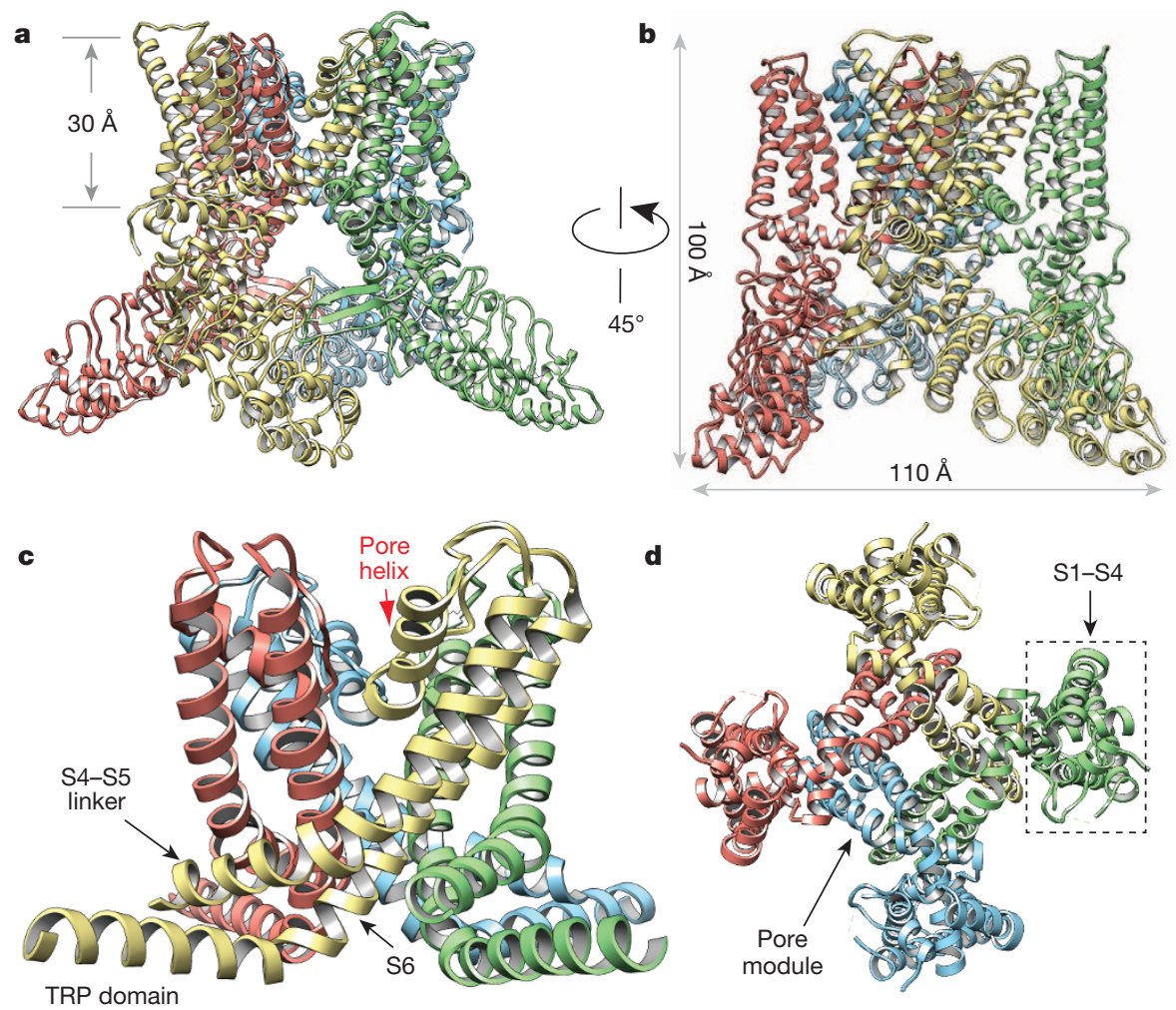

Figure 2 TRPV1 and VGICs share similar four-fold symmetric

architecture. a-d, Ribbon diagram of TRPV1 atomic model with each of the four identical subunits colour-coded, showing views from side (a and $\mathbf{b}$ ).

The dimensions of the channel and the length of the membrane-spanning helices are indicated. The dimensions of the TRPV1 tetramer are $100 \AA \times 110 \AA \times 110 \AA$, as compared with $135 \AA \times 95 \AA \times 95 \AA$ for the rat

after S6 (Fig. 2c). This $\alpha$-helix encompasses the first two-thirds of the TRP domain, after which the structure transitions to a random coil (Fig. 3b). An invariant tryptophan (W697) near the middle of the TRP domain forms a hydrogen bond with the main-chain carbonyl oxygen of F559 at the beginning of the S4-S5 linker (Extended Data Fig. 10c). Interestingly, gain-of-function mutations at this equivalent tryptophan in TRPV3 underlie a congenital disorder, Olmsted syndrome, in humans ${ }^{31}$. Moreover, charged side chains within the TRP domain are located on the side of the helix facing the cytoplasm, where they interact with the
$\mathrm{K}_{\mathrm{V}} 1.2$ potassium channel ${ }^{5}$. c, Ribbon diagram focusing in on side view of S5-P-S6 pore with TRP domains. d, Bottom view focusing on transmembrane core, including S1-S4, S5-P-S6 and TRP domains. Note that S1-S4 domains flank and interact with S5-P-S6 pore modules from adjacent subunit, reminiscent of VGIC architecture.

pre-S1 helix through hydrogen bonding and salt bridging (Fig. 4e and Extended Data Fig. 10c, d). Taken together, these observations are consistent with a role for the TRP helix as a point of structural integration that facilitates allosteric coupling between channel domains.

In VGICs, the S4-S5 linker has a critical role in coupling the movement of the S1-S4 voltage sensor to gating of the pore ${ }^{5-8,32}$. Gain-offunction mutations within the S4-S5 linker or S5 helix of TRPV4 enhance basal open probability and result in skeletal dysplasia syndromes ${ }^{33}$. Interestingly, a cation $-\pi$ interaction can be seen between residues at

a
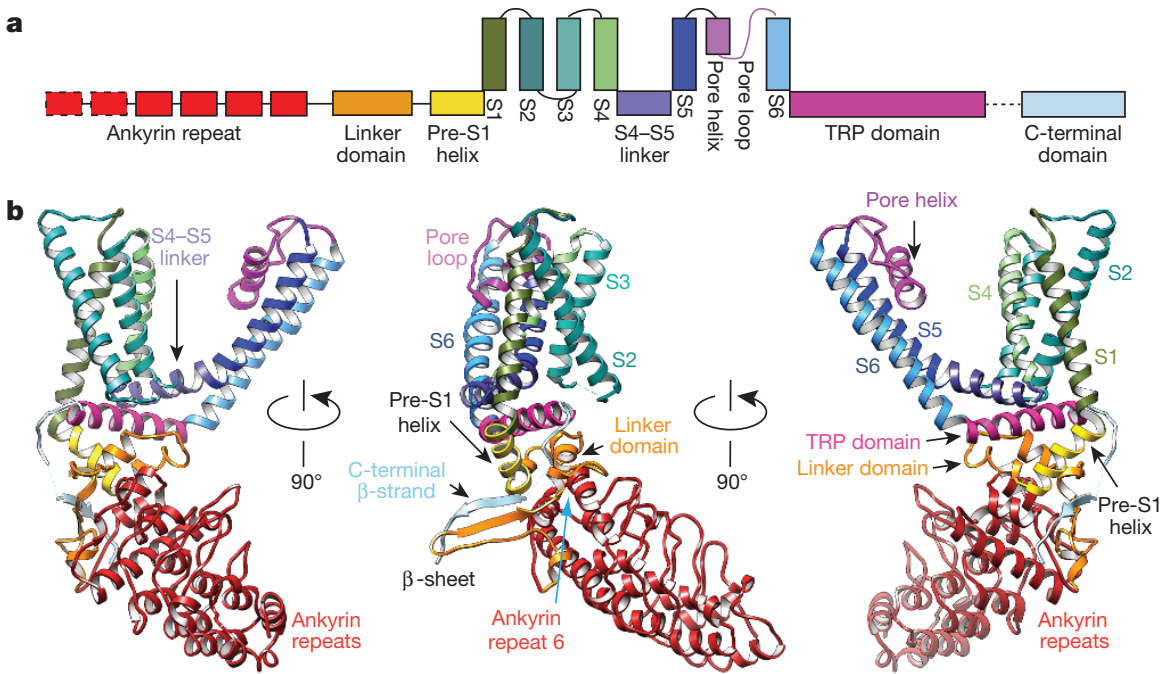

Figure 3 Structural details of a single TRPV1 subunit. a, Linear diagram depicting major structural domains in a TRPV1 subunit, colour coded to match ribbon diagrams below. Dashed boxes denote regions for which density was not

observed (first two ankyrin repeats) or where specific residues could not be definitively assigned (C-terminal $\beta$-strand). $\mathbf{b}$, Ribbon diagrams showing three different views of a TRPV1 monomer denoting specific domains. 

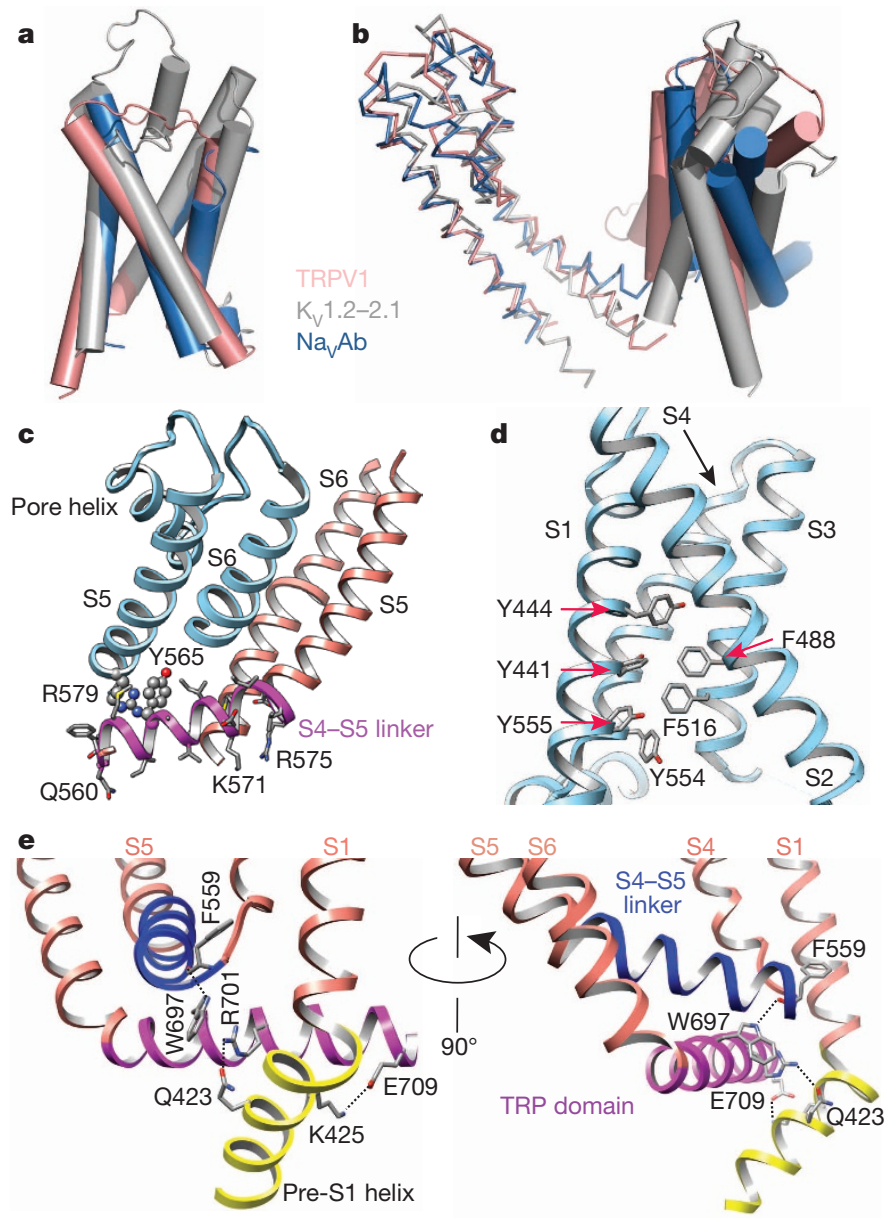

Figure $4 \mid$ Unique structural features of TRPV1. a, Alignment of S1-S4 transmembrane domains from TRPV1 (salmon), $\mathrm{K}_{\mathrm{V}} 1.2-2.1$ chimaera (PDB 2R9R; grey) and $\mathrm{Na}_{\mathrm{V}} \mathrm{Ab}$ (PDB 3RVY; blue) show substantial overlap. b. When S5-P-S6 pore regions are aligned, the S1-S4 domains show differential relative orientations. $c$, The S4-S5 linker is an amphipathic $\alpha$-helix whose charged surface faces the cytosol. Potential cation $-\pi$ interactions between Y565 in the S4-S5 linker and R579 from S5 of the adjacent subunit are highlighted. Mutations of cognate residues in TRPV4 render the channel constitutively active and cause skeletal dysplasia. d, Aromatic side chains from S1, S3 and S4 helices create a hydrophobic interior in the S1-S4 domains, in contrast to the charged environment observed in VGICs. e, Two different views highlight interactions between TRP domain and S4-S5 linker and pre-S1 helix. Interactions (that is, hydrogen bonds and salt bridge) are indicated by dashed lines.

equivalent positions in TRPV1, namely Y565 in the S4-S5 linker of one subunit and R579 in S5 of a neighbouring subunit (Fig. 4c and Extended Data Fig. 10a). Thus, in addition to covalent intra-subunit interaction between the S4-S5 linker and S5-P-S6 pore region, functional coupling may also be achieved through weak trans-subunit interactions.

\section{The pore and ion permeation pathway}

The outer pore region of TRPV1 is wide open compared to $\mathrm{K}_{\mathrm{V}}$ channels, with a broad funnel-like structure that probably enhances accessibility to both small and large pharmacophores (Fig. 5a, b). Indeed, as described in the accompanying study ${ }^{29}$, the uppermost regions of the pore loop proximal to S5 and S6 form a binding site for spider toxins. Further down the central canal, we see a short selectivity filter $\left({ }^{643} \mathrm{GMGD}^{646}\right)$ in which backbone carbonyls or side chains point into the central pathway (Fig. 5a-e). TRPV1 and related subtypes are highly calcium permeable $e^{9,34}$ and molecular-modelling studies predict a selectivity filter diameter of $\sim 6 \AA^{35,36}$. In our structure, we see a restriction point of $4.6 \AA$ between diagonally opposed carbonyl oxygens at G643 (Fig. 5a-c, e), suggesting that the selectivity filter is in a non-conducting state, reminiscent of inactivated bacterial $\mathrm{Na}_{V} \mathrm{Rh}$ channels ${ }^{8}$. Indeed, as we show in the accompanying study ${ }^{29}$, the selectivity filter diameter increases substantially when TRPV1 is in its activated state. The overall arrangement that we see for TRPV1 - namely, a wide outer pore with a short selectivity filtermore closely resembles that of bacterial $\mathrm{Na}_{\mathrm{V}}$ channels than $\mathrm{K}_{\mathrm{V}}$ channels (Extended Data Fig. 10e, f) ${ }^{5-8}$. However, TRPV1 lacks hydrogen bonding within and between adjacent pore helices, in contrast to $\mathrm{Na}_{\mathrm{v}}$ channels, in which such elaborate interactions are believed to impart rigidity to the selectivity filter ${ }^{7}$. This more flexible architecture, together with a short selectivity filter, may underlie the phenomenon of pore dilation seen in several TRP channels, including TRPV1, whereby prolonged or repetitive activation renders the channel permeable to large organic cations ${ }^{21,37}$. Interestingly, when compared with TRPV1-4, TRPV5 and TRPV6 subtypes bear distinct sequences at the selectivity filter (that is, T-V/I-I-D versus G-M/L-G-D/E), as well as the pore helix, suggesting a different architecture of the outer pore domain (Extended Data Fig. 2). This divergence may underlie the fact that TRPV5 and TRPV6 exhibit more pronounced selectivity for calcium over monovalent cations (permeability $(P)_{\mathrm{Ca}}{ }^{2+} / P_{\mathrm{Na}}{ }^{+}>100$ ) compared to other TRPV subtypes $\left(P_{\mathrm{Ca}}{ }^{2+} / P_{\mathrm{Na}}{ }^{+} \approx 3-10\right)$ (ref. 34).

Continuing down the pore, we see a constriction site in which I679 in $\mathrm{S} 6$ helices from each subunit come together to form a hydrophobic seal measuring $5.3 \AA$ between side chains, thus constituting the most constricted point in the lower gate (Fig. $5 \mathrm{a}-\mathrm{c}$, g). Modelling studies, together with substituted cysteine accessibility analysis, have previously implicated I679, Y671 and L681 as candidates for this position ${ }^{35,38}$. Our structure indicates that side chains of Y671 are located $\sim 10 \AA$ above the narrowest point formed by I679 (Fig. 5a, f), and that the side chain of L681 points away from the ion conduction pathway, making Y671 and L681 unlikely major contributors to the lower gate. Of note, TRPV subtypes exhibit high sequence similarity throughout the $\$ 6$ helix, and all contain an isoleucine at the position equivalent to I679 in TRPV1 (Extended Data Fig. 2), suggesting a similar architecture in the lower gate. Whereas the narrowest constriction site in bacterial KcsA and $\mathrm{Na}_{\mathrm{V}} \mathrm{Ab}$ channels is located at the apex of the inverted teepee ${ }^{7,39}$, the I679 restriction point is higher in the TRPV1 permeation pathway, sitting $\sim 10 \AA$ above the S6 bundle crossing. We believe that the structure shown here represents TRPV1 in the closed state, a conclusion that is validated by comparison with structures representing agonistbound states of the channel described in the accompanying study ${ }^{29}$.

\section{Ankyrin repeats form an assembly domain}

A characteristic feature of many TRP channels is the presence of ankyrin repeats within the cytoplasmic $\mathrm{N}$ terminus ${ }^{40}$. In our structure, residues in the atypically long finger 3 and inner helices of ankyrin repeats 3 and 4 from one subunit interact with a three-stranded antiparallel $\beta$-sheet formed by the ARD-S1 linker region (K368-D383) and C terminus of an adjacent subunit, packing the cytosolic part of the channel together (Fig. 6). Moreover, the $\beta$-sheet structure tethers cytoplasmic N- and $\mathrm{C}$-terminal domains together within the same subunit, reminiscent of G-protein-coupled inwardly rectifying potassium channels, in which $\mathrm{N}$ - and $\mathrm{C}$ termini interact through a short parallel $\beta$-sheet ${ }^{41,42}$. Interestingly, two residues (G375 and P376) that produce the sharp turn connecting the $\mathrm{N}$-terminal $\beta$-strands are invariant among all TRPV subtypes (Extended Data Fig. 2), supporting the proposed functional importance of the $\beta$-sheet in subunit structure and assembly.

In the TRPV1 ARD crystal structure, ATP is bound within the concave surface formed by ankyrin repeats $1-3$, and has been proposed to both stabilize the ARD fold and regulate $\mathrm{Ca}^{2+}$-calmodulin-dependent desensitization $^{24}$. Moreover, a number of mutations that render TRPV1 or other TRPV channels constitutively active lie in the vicinity of the proposed ATP binding site $e^{22,43}$, further indicating that the ARD is an important locus for channel modulation. Our structural data suggest that modulation may also involve perturbation of subunit-subunit interactions. At the same time, the ARD surface forms an extensive cytoplasmic 
a
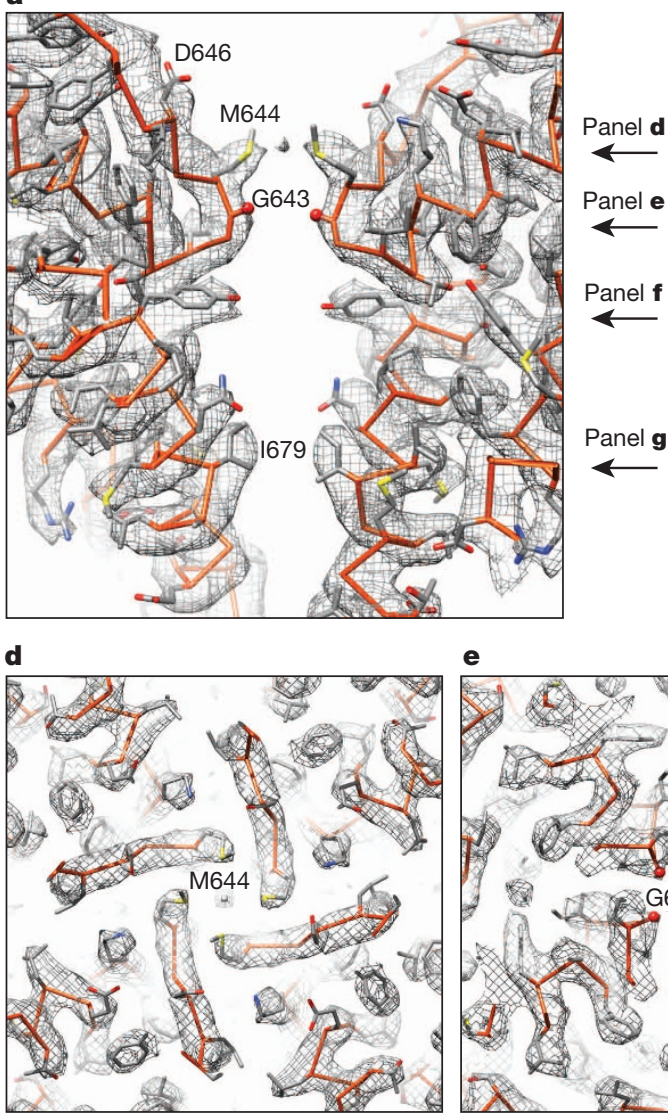

e

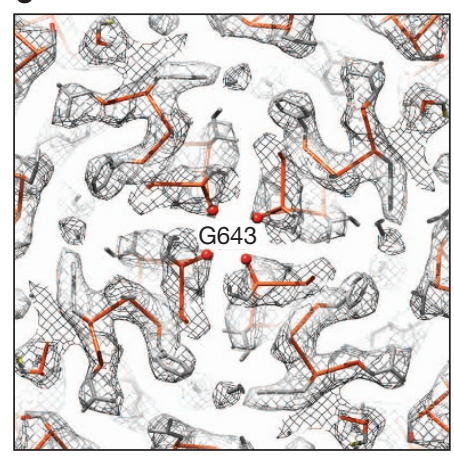

Figure $5 \mid$ The ion permeation pathway of TRPV1. a, Cryo-EM densities of the pore in longitudinal cross section are superimposed on an atomic model. Only two diagonally opposed subunits are shown for clarity. Several residues along the pore are labelled for orientation. Arrows denote positions of density maps for horizontal cross sections shown in panels $\mathbf{d}-\mathbf{g}$, as indicated. b, Solvent-accessible pathway along the pore mapped using the HOLE

skirt, much of which remains available for interaction with other, as yet unidentified cellular factors. Whether and how these regions contribute to channel regulation may be revealed by structures of TRPV channels in complex with physiological modulators that target this domain.

\section{Concluding remarks}

TRP channels represent one of the last major ion channel families to yield to structure determination at the atomic level. Obtaining crystals c

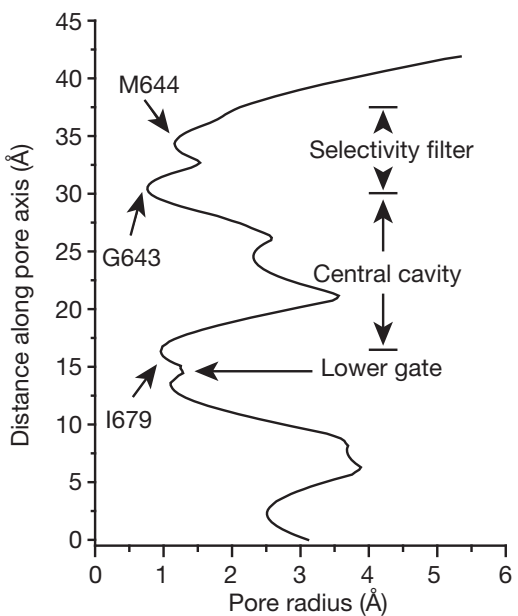

g

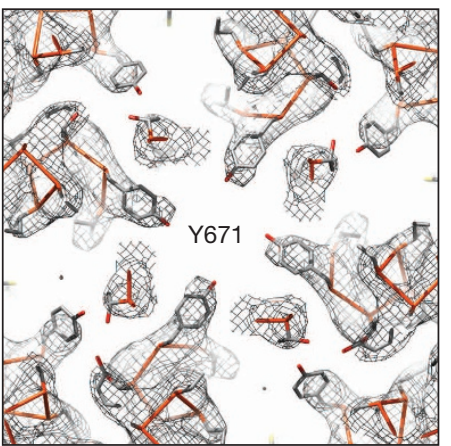

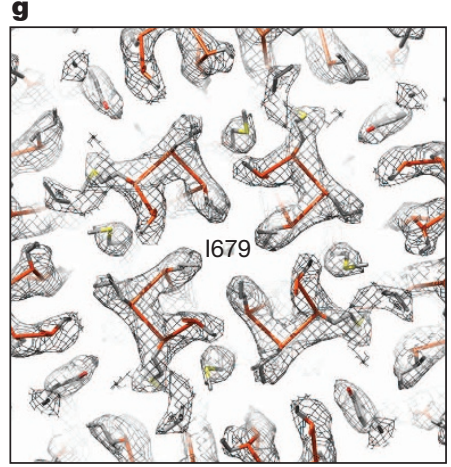

program. Residues located at the selectivity filter and lower gate are rendered as sticks. c, radius of the pore calculated with program HOLE. d-g, Cryo-EM densities of several residues along the pore are superimposed on the atomic model; all panels represent views along the four-fold axis, showing residues from each subunit of the homotetrameric channel.

for membrane proteins, particularly of mammalian origin, is generally challenging, perhaps especially so for TRP channels, which respond to diverse stimuli (chemical and physical) and are therefore believed to be conformationally dynamic. If so, then this represents an additional obstacle to coaxing these proteins into forming well-ordered crystal lattices required for X-ray or electron crystallographic analysis. We have circumvented this problem by taking advantage of recent technological breakthroughs in single-particle cryo-EM to gain high-resolution
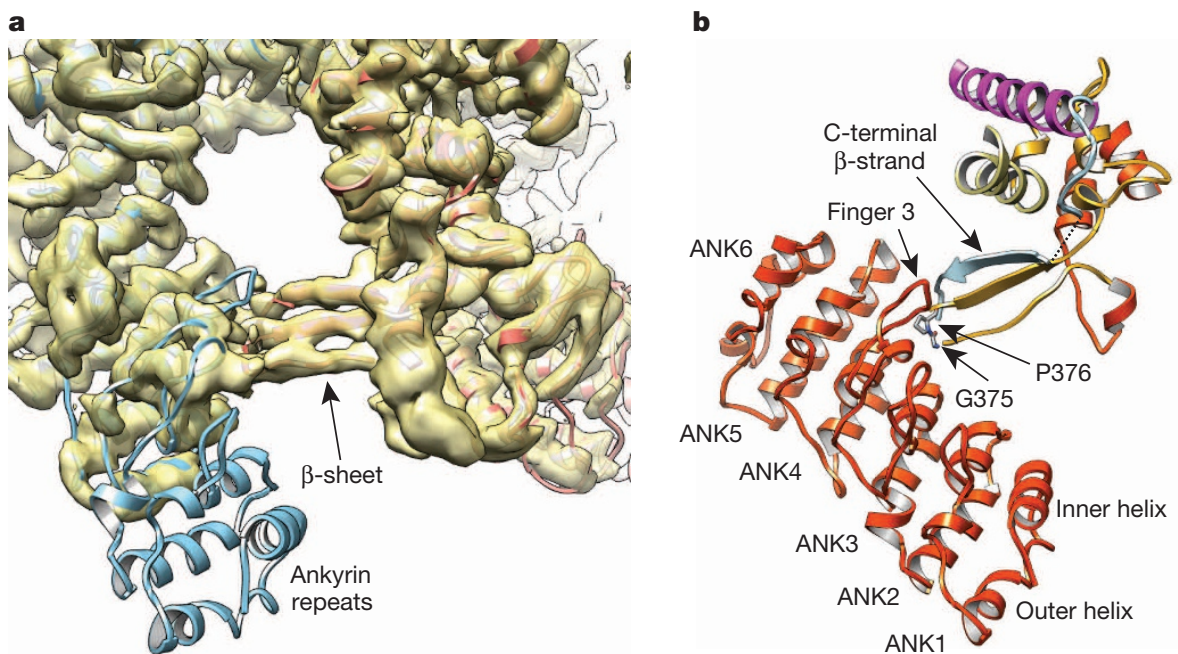

Figure 6 | Cytosolic interactions mediated by ARDs. a, Cryo-EM density is well fitted by crystal structure of TRPV1 ankyrin repeats 3-6 (PDB 2PNN) as a rigid body. Ankyrin repeats 1 and 2 are not observed, presumably owing to high flexibility. b. Finger 3 and inner helices from ankyrin repeat 3 and 4 on the concave surface of the ARD interact with $\beta$-strands from the linker and $\mathrm{C}$ terminus of an adjacent subunit. 
structural information of purified TRP channels under conditions that do not require absolute conformational homogeneity (supported by the fact that only a subset of the data set was used to generate the final 3D reconstruction), or large amounts of material. We expect that this approach will be particularly powerful for analysing heterogeneous populations of channels with the goal of identifying and characterizing different conformational states en route to gating, or in complex with agonists, drugs or other modulators. Indeed, we provide a demonstration of this in the accompanying study ${ }^{29}$.

\section{METHODS SUMMARY}

A minimal-functional rat TRPV1 construct was cloned into a modified BacMam vector (Invitrogen) containing an N-terminal fusion cassette (Kozac-MBP-tobacco etch virus (TEV) protease site) for purification on amylose resin. TRPV1 protein was expressed in HEK293S $\mathrm{GnTI}^{-}$cells grown in suspension at $37^{\circ} \mathrm{C}$. Cells were collected $48 \mathrm{~h}$ after transduction for preparation of crude membrane and subsequent protein purification, as described ${ }^{10}$. Negative-stain EM and cryo-EM were carried out following established protocols ${ }^{15,44}$. At $3.4 \AA$ resolution, the cryo-EM map was of sufficient quality for de novo atomic model building. A poly-alanine model was first built in Coot and amino acid assignment subsequently achieved based mainly on the clearly defined side-chain densities of bulky residues such as Phe, Tyr and Trp, as well as some Arg and Lys residues.

Online Content Any additional Methods, Extended Data display items and Source Data are available in the online version of the paper; references unique to these sections appear only in the online paper.

\section{Received 24 July; accepted 30 October 2013.}

1. Ramsey, I. S., Delling, M. \& Clapham, D. E. An introduction to TRP channels. Annu. Rev. Physiol. 68, 619-647 (2006).

2. Venkatachalam, K. \& Montell, C. TRP channels. Annu. Rev. Biochem. 76, 387-417 (2007).

3. Nilius, B. \& Owsianik, G. Transient receptor potential channelopathies. Pflugers Arch. 460, 437-450 (2010).

4. Nilius, B. etal. Gating of TRP channels: a voltage connection? J. Physiol. (Lond.) 567, 35-44 (2005)

5. Long, S. B., Campbell, E. B. \& Mackinnon, R. Crystal structure of a mammalian voltage-dependent Shaker family K ${ }^{+}$channel. Science 309, 897-903 (2005).

6. Long, S. B., Tao, X., Campbell, E. B. \& MacKinnon, R. Atomic structure of a voltagedependent $\mathrm{K}^{+}$channel in a lipid membrane-like environment. Nature $\mathbf{4 5 0}$ 376-382 (2007)

7. Payandeh, J., Scheuer, T., Zheng, N. \& Catterall, W. A. The crystal structure of a voltage-gated sodium channel. Nature 475, 353-358 (2011).

8. Zhang, X. et al. Crystal structure of an orthologue of the NaChBac voltage-gated sodium channel. Nature 486, 130-134 (2012).

9. Caterina, M. J. etal. The capsaicin receptor: a heat-activated ion channel in the pain pathway. Nature 389, 816-824 (1997).

10. Cao, E., Cordero-Morales, J. F., Liu, B., Qin, F. \& Julius, D. TRPV1 channels are intrinsically heat sensitive and negatively regulated by phosphoinositide lipids. Neuron 77, 667-679 (2013).

11. Yao, J., Liu, B. \& Qin, F. Kinetic and energetic analysis of thermally activated TRPV1 channels. Biophys. J. 99, 1743-1753 (2010).

12. Brederson, J. D., Kym, P. R. \& Szallasi, A. Targeting TRP channels for pain relief. Eur. J. Pharmacol. 716, 61-76 (2013)

13. Julius, D. TRP channels and pain. Annu. Rev. Cell Dev. Biol. 29, 355-384 (2013).

14. Bai, X. C., Fernandez, I. S., McMullan, G. \& Scheres, S. H. Ribosome structures to near-atomic resolution from thirty thousand cryo-EM particles. eLife 2, e00461 (2013).

15. Li, X. et al. Electron counting and beam-induced motion correction enable nearatomic-resolution single-particle cryo-EM. Nature Methods 10, 584-590 (2013).

16. Yu, X., Jin, L. \& Zhou, Z. H. $3.88 \AA$ Atructure of cytoplasmic polyhedrosis virus by cryo-electron microscopy. Nature 453, 415-419 (2008).

17. Zhang, X., Jin, L., Fang, Q., Hui, W. H. \& Zhou, Z. H. 3.3 A cryo-EM structure of a nonenveloped virus reveals a priming mechanism for cell entry. Cell 141, 472-482 (2010).

18. Mio, K. et al. The TRPC3 channel has a large internal chamber surrounded by signal sensing antennas. J. Mol. Biol. 367, 373-383 (2007).

19. Moiseenkova-Bell, V. Y., Stanciu, L. A., Serysheva, I. I., Tobe, B. J. \& Wensel, T. G. Structure of TRPV1 channel revealed by electron cryomicroscopy. Proc. Natl Acad. Sci. USA 105, 7451-7455 (2008).

20. Shigematsu, H., Sokabe, T., Danev, R., Tominaga, M. \& Nagayama, K. A 3.5-nm structure of rat TRPV4 cation channel revealed by Zernike phase-contrast cryoelectron microscopy. J. Biol. Chem. 285, 11210-11218 (2010).

21. Chung, M. K., Guler, A. D. \& Caterina, M. J. TRPV1 shows dynamic ionic selectivity during agonist stimulation. Nature Neurosci. 11, 555-564 (2008).

22. Myers, B. R., Bohlen, C. J. \& Julius, D. A yeast genetic screen reveals a critical role for the pore helix domain in TRP channel gating. Neuron 58, 362-373 (2008).
23. Scheres, S. H. \& Chen, S. Prevention of overfitting in cryo-EM structure determination. Nature Methods 9, 853-854 (2012).

24. Lishko, P. V., Procko, E., Jin, X., Phelps, C. B. \& Gaudet, R. The ankyrin repeats of TRPV1 bind multiple ligands and modulate channel sensitivity. Neuron $\mathbf{5 4}$, 905-918 (2007)

25. Catterall, W. A. Ion channel voltage sensors: structure, function, and pathophysiology. Neuron 67, 915-928 (2010)

26. Swartz, K. J. Sensing voltage across lipid membranes. Nature 456, 891-897 (2008).

27. Boukalova, S. Marsakova, L. Teisinger, J. \& Vlachova, V. Conserved residues within the putative S4-S5 region serve distinct functions among thermosensitive vanilloid transient receptor potential (TRPV) channels. J. Biol. Chem. 285, 41455-41462 (2010).

28. Boukalova, S., Teisinger, J. \& Vlachova, V. Protons stabilize the closed conformation of gain-of-function mutants of the TRPV1 channel. Biochim. Biophys. Acta 1833, 520-528 (2013)

29. Cao, E., Liao, M., Cheng, Y. \& Julius, D. TRPV1 structures in distinct conformations reveal mechanisms of activation. Nature http://dx.doi.org/10.1038/nature12823 (this issue).

30. Latorre, R., Zaelzer, C. \& Brauchi, S. Structure-functional intimacies of transient receptor potential channels. Q. Rev. Biophys. 42, 201-246 (2009).

31. Lin, Z. et al. Exome sequencing reveals mutations in TRPV3 as a cause of Olmsted syndrome. Am. J. Hum. Genet. 90, 558-564 (2012).

32. Long, S. B., Campbell, E. B. \& Mackinnon, R. Voltage sensor of Kv1.2: structural basis of electromechanical coupling. Science 309, 903-908 (2005).

33. Loukin, S., Su, Z. \& Kung, C. Increased basal activity is a key determinant in the severity of human skeletal dysplasia caused by TRPV4 mutations. PLOS ONE 6, e19533 (2011)

34. Owsianik, G., Talavera, K., Voets, T. \& Nilius, B. Permeation and selectivity of TRP channels. Annu. Rev. Physiol. 68, 685-717 (2006).

35. Susankova, K., Ettrich, R., Vyklicky, L., Teisinger, J. \& Vlachova, V. Contribution of the putative inner-pore region to the gating of the transient receptor potential vanilloid subtype 1 channel (TRPV1). J. Neurosci. 27, 7578-7585 (2007).

36. Voets, T., Janssens, A., Droogmans, G. \& Nilius, B. Outer pore architecture of a Ca2 ${ }^{+}$-selective TRP channel. J. Biol. Chem. 279, 15223-15230 (2004).

37. Binshtok, A. M., Bean, B. P. \& Woolf, C. J. Inhibition of nociceptors by TRPV1 mediated entry of impermeant sodium channel blockers. Nature $449,607-610$ (2007).

38. Salazar, H. et al. Structural determinants of gating in the TRPV1 channel. Nature Struct. Mol. Biol. 16, 704-710 (2009).

39. Doyle, D. A. et al. The structure of the potassium channel: molecular basis of $\mathrm{K}^{+}$conduction and selectivity. Science 280, 69-77 (1998).

40. Gaudet, R. A primer on ankyrin repeat function in TRP channels and beyond. Mol. Biosyst. 4, 372-379 (2008).

41. Inanobe, A., Matsuura, T., Nakagawa, A. \& Kurachi, Y. Structural diversity in the cytoplasmic region of $\mathrm{G}$ protein-gated inward rectifier $\mathrm{K}^{+}$channels. Channels (Austin) 1, 39-45 (2007).

42. Nishida, M. \& MacKinnon, R. Structural basis of inward rectification: cytoplasmic pore of the G protein-gated inward rectifier GIRK1 at $1.8 \AA$ A resolution. Cell 111, 957-965 (2002).

43. Inada, H., Procko, E., Sotomayor, M. \& Gaudet, R. Structural and biochemical consequences of disease-causing mutations in the ankyrin repeat domain of the human TRPV4 channel. Biochemistry 51, 6195-6206 (2012)

44. Booth, D. S., Avila-Sakar, A. \& Cheng, Y. Visualizing proteins and macromolecular complexes by negative stain EM: from grid preparation to image acquisition. J. Vis. Exp. 58, 3227 (2011)

Acknowledgements We thankX. Li for assistance with data acquisition using TF30 Polara and K2 Summit camera, S. Zhou and D. King for help with protein microsequencing and J.P Armache, C. Bohlen, J. Cordero-Morales and J. Osteen for discussion and reading of the manuscript. This work was supported by grants from the National Institutes of Health (R01GM098672 and S1ORR026814 to Y.C. and R01NS065071 and R01NS047723 to D.J.), the National Science Foundation (DBI-0960271 to D. Agard and Y.C.) and the University of California, San Francisco Program for Breakthrough Biomedical Research (Y.C.). E.C. was a fellow of the Damon Runyon Cancer Research Foundation.

Author Contributions All authors designed experiments. E.C. expressed and purified al protein samples used in this work and performed all functional studies. M.L. carried out all cryo-EM experiments, including data acquisition and processing. E.C. built the atomic model on the basis of cryo-EM maps. All authors analysed data and wrote the manuscript.

Author Information 3D cryo-EM density map of TRPV1 complexes without low-pass filter and amplitude modification have been deposited in the Electron Microscopy Data Bank under the accession number EMD-5778 (TRPV1). Particle images related to this entry are available for download at http://www.ebi.ac.uk/ ardan/aspera/ em-aspera-demo.html with identification no. 10005. The coordinates of atomic model of TRPV1 have been deposited in the Protein Data Bank under the accession number 3J5P. Reprints and permissions information is available at www.nature.com/reprints. The authors declare no competing financial interests. Readers are welcome to comment on the online version of the paper. Correspondence and requests for materials should be addressed to D.J. (david.julius@ucsf.edu) or Y.C. (ycheng@ucsf.edu). 


\section{METHODS}

Protein expression and purification. A BacMam vector was generated from pFastBac1 (Invitrogen) to direct protein expression in mammalian cells after baculovirus transduction ${ }^{45}$. In brief, the polyhedron promoter $\left(\mathrm{P}_{\mathrm{PH}}\right)$ in pFastBacl was replaced with a mammalian cell active promoter $\left(\mathrm{P}_{\mathrm{CMV}}\right)$, immediately followed by an N-terminal fusion cassette (Kozac-MBP-tobacco etch virus (TEV) protease site) for affinity purification with amylose resin (New England Biolabs). A minimal functional rat TRPV1 construct, composed of amino acids 110 to 603 and 627 to 764 , was cloned into this modified BacMam vector, and recombinant baculoviruses obtained following the manufacturer's protocol (Bac-to-Bac expression system, Invitrogen). For protein expression, HEK293S $\mathrm{GnTI}^{-}$cells $^{46}$, grown in suspension at $37^{\circ} \mathrm{C}$ in an orbital shaker, were transduced when cell density reached $\sim 2 \times 10^{6}$ per $\mathrm{ml}$. Sodium butyrate was added to the culture $24 \mathrm{~h}$ after transduction at a final concentration of $10 \mathrm{mM}$ to boost protein expression, and cells collected $48 \mathrm{~h}$ after transduction for preparation of crude membrane and subsequent protein purification, as described ${ }^{10}$ with slight modification. TRPV1 channel was eluted from amylose resin with buffer composed of $150 \mathrm{mM} \mathrm{NaCl}, 2 \mathrm{mM}$ tris(2-carboxyethyl)phosphine (TCEP), 10\% glycerol, $20 \mathrm{mM}$ HEPES, $0.5 \mathrm{mM}$ DDM, $0.1 \mathrm{mg} \mathrm{ml}^{-1}$ soybean lipids, and $20 \mathrm{mM}$ maltose, then incubated with TEV protease for $4 \mathrm{~h}$ at $4{ }^{\circ} \mathrm{C}$. The cleaved protein sample was then mixed with amphipols at $1: 3(\mathrm{w} / \mathrm{w})$ with gentle agitation for another $4 \mathrm{~h}$. Detergent was removed with Bio-Beads SM-2 ( $4{ }^{\circ} \mathrm{C}$ overnight, $15 \mathrm{mg}$ per $1 \mathrm{ml}$ channel/detergent/amphipols mixture). Bio-beads were then removed over a disposable polyprep column, and eluent cleared by centrifugation before further separation on a Superdex 200 column in buffer composed of $150 \mathrm{mM} \mathrm{NaCl}, 20 \mathrm{mM}$ HEPES, $2 \mathrm{mM}$ TCEP, $\mathrm{pH}$ 7.4. The peak corresponding to tetrameric TRPV1 channels was collected for analysis by cryo-EM.

Cell imaging and electrophysiology. Forty-eight hours after transduction, HEK293S $\mathrm{GnTI}^{-}$cells were loaded with Fura-2-acetoxymethyl ester in physiologic Ringer's buffer ( $140 \mathrm{mM} \mathrm{NaCl}, 5 \mathrm{mM} \mathrm{KCl}, 2 \mathrm{mM} \mathrm{CaCl}_{2}, 2 \mathrm{mM} \mathrm{MgCl}_{2}, 10 \mathrm{mM}$ HEPES, pH 7.4) for ratiometric calcium imaging. Currents were recorded at room temperature in the whole-cell patch-clamp configuration. The intracellular solution contained $150 \mathrm{mM} \mathrm{NaCl}, 10 \mathrm{mM}$ HEPES, $10 \mathrm{mM}$ EGTA, pH 7.4. DkTx and capsaicin were freshly diluted into this buffer and applied via an in-line perfusion system (Automatic Scientific). To obtain proton-evoked current, buffer consisting of $150 \mathrm{mM}$ $\mathrm{NaCl}, 10 \mathrm{mM}$ sodium acetate, 10 mM EGTA, pH 5.0 was perfused onto TRPV1expressing cells. Purified TRPV1 protein was also reconstituted into soybean lipid liposomes, and activation by capsaicin or heat examined by patch-clamp analysis, as described ${ }^{10}$. To estimate ion permeability ratios, the intracellular solution contained: $150 \mathrm{mM} \mathrm{NaCl}, 10 \mathrm{mM}$ HEPES, $10 \mathrm{mM}$ EDTA, pH 7.4, and extracellular solutions contained: (A) $150 \mathrm{mM} \mathrm{KCl}, 10 \mathrm{mM}$ HEPES, $10 \mathrm{mM}$ EDTA, pH 7.4; (B) $150 \mathrm{mM}$ CsCl, 10 mM HEPES, 10 mM EDTA, pH 7.4; (C) $110 \mathrm{mM} \mathrm{CaCl}_{2}, 10 \mathrm{mM}$ HEPES, pH 7.4. Resiniferatoxin, capsaicin and DkTx were diluted in appropriate solution immediately before use, and applied to the TRPV1-expressing cell using an in-line perfusion system

EM data acquisition. Detergent solubilized TRPV1 particles were monodispersed as assessed by negative-stain EM (Extended Data Fig. 3a, b), enabling us to obtain a $3 \mathrm{D}$ reconstruction by random conical tilt (Extended Data Fig. 3c, d), which served as an initial model for cryo-EM 3D refinement. Grids for negative-stain EM were prepared following the established protocol $^{44}$. Specifically, $2.5 \mu$ l of purified TRPV1 was applied to glow-discharged EM grids covered by a thin layer of continuous carbon film and was stained with $0.75 \%(\mathrm{w} / \mathrm{v})$ uranyl formate. Negatively stained EM grids were imaged on a Tecnai T12 microscope (FEI Company) operated at $120 \mathrm{kV}$. Images were recorded at a nominal magnification of $67,000 \times$ using a $4 \mathrm{k} \times 4 \mathrm{k}$ charge-coupled device camera (UltraScan 4000, Gatan), corresponding to a pixel size of $1.73 \AA$ per pixel on the specimen. Tilt pair images for random conical tilt $3 \mathrm{D}$ reconstruction were manually recorded at $50^{\circ}$ and $0^{\circ}$.

For cryo-EM, detergent was replaced by amphipols ${ }^{47,48}$, in which purified TRPV1 remained stable and monodispersed (Extended Data Fig. 3e-g). $2 \mu$ l of purified TRPV1 sample at a concentration of $\sim 0.3 \mathrm{mg} \mathrm{ml}^{-1}$ was applied to a glowdischarged Quantifoil holey carbon grid (1.2 $\mu \mathrm{m}$ hole size, $400 \mathrm{mesh}$ ), blotted inside a Vitrobot Mark III (FEI Company) using 6-s blotting time with $90 \%$ humidity, and then plunge-frozen in liquid ethane cooled by liquid nitrogen. Cryo-EM images were collected at liquid nitrogen temperature on a Tecnai TF20 electron microscope (FEI) operated at $200 \mathrm{kV}$ using a CT3500 side entry holder (Gatan), following the low-dose procedure. On the TF20 microscope, images were recorded at a nominal magnification of $80,000 \times$ using a phosphor scintillator based TemF816 8K $\times 8 \mathrm{~K}$ CMOS camera (TVIPS GmbH), corresponding to a pixel size of $0.9 \AA$ per pixel on the specimen. Images were recorded with a defocus in the range from 1.5 to $3.5 \mu \mathrm{m}$ (Extended Data Fig. 4). We determined a 3D reconstruction with $\mathrm{C} 4$ symmetry to an overall resolution of $8.8 \AA$, using gold-standard FSC $=0.143$ criteria $^{23}$ (Extended Data Fig. 5a). In this density map, transmembrane $\alpha$-helices are clearly resolved. The shape of the cytoplasmic domain is clearly defined, but secondary structural features are not well resolved (Extended Data Figs $5 b-f)$, probably indicating greater flexibility of this region.

Another data set of frozen hydrated TRPV1 particles were collected on a TF30 Polara electron microscope (FEI Company) operated at $300 \mathrm{kV}$. It is equipped with a K2 Summit direct electron detector camera (Gatan). Images were recorded using super-resolution counting mode following an established protocol ${ }^{15}$. Specifically, images from TF30 were recorded at a nominal magnification of $31,000 \times$, corresponding to a calibrated super resolution pixel size of $0.6 \AA$ per pixel on the specimen. The dose rate on the camera was set to be $\sim 8$ counts (corresponding to $\sim 9.9$ electron) per physical pixel per second. The total exposure time was $6 \mathrm{~s}$, leading to a total accumulated dose of 41 electrons per $\AA^{2}$ on the specimen. Each image was fractionated into 30 subframes, each with an accumulation time of $0.2 \mathrm{~s}$ per frame. All dose-fractionated cryo-EM images were recorded using a semiautomated acquisition program UCSFImage4 (written by X. Li). Images were recorded with a defocus in a range from 1.5 to $3.0 \mu \mathrm{m}$.

Image processing. SamViewer, an interactive image analysis program written in wxpython, was used for all 2D image display and particle picking. Negative-stain EM images were $2 \times 2$ binned for manual particle picking. Defocus was determined using CTFFIND and CTFTILT ${ }^{49}$. Individual particles were cut out and normalized to have a mean of 0 and a standard deviation of 1 . For 2D classification, particles were first corrected for contrast transfer function (CTF) by flipping the phase using 'ctfapply' (written by X. Li), and subjected to 10 cycles of correspondence analysis, $k$-means classification and multi-reference alignment (MRA), using SPIDER operations 'CA S', 'CL KM' and 'AP SH'50. For random conical tilt (RCT) $3 \mathrm{D}$ reconstruction, SamViewer was used for picking particles from the tilt-pair images, as well as determination of the tilting axes and angles. After 2D classification of untilted particles, RCT 3D reconstructions of each 2D class were calculated using FREALIGN ${ }^{51}$

Low-dose images of frozen hydrated TRVP1 collect on TF20 were binned $2 \times 2$, resulting with a pixel size of $1.9 \AA$, for image processing. For particle picking and $2 \mathrm{D}$ classification, images were $2 \times 2$ binned further to a pixel size of $3.8 \AA$. Dosefractionated super-resolution image stacks of frozen hydrated TRPV1 images collected using K2 Summit camera were first binned $2 \times 2$ resulting with a pixel size of $1.2 \AA$ for motion correction and further image processing. After motion correction $^{15}$, a sum of all subframes in each image stack was used for further processing. Particle picking and 2D classification used $6 \times 6$ binned images ( $3.6 \AA$ per pixel), and 3D classification used $4 \times 4$ binned images $(2.4 \AA$ per pixel). Final 3D reconstruction was calculated from $2 \times 2$ binned images (1.2 A per pixel). Image binning was calculated using Fourier cropping. First, $\sim 2,000$ particles were picked interactively and classified into $\sim 102 \mathrm{D}$ classes using the same classification procedure described above. Then, for each micrograph, the entire image was cut into a set of overlapping small images with a window of $64 \times 64$ pixels, all windowed images were subject to MRA against the $2 \mathrm{D}$ class averages generated from manually selected particles (implemented in a python script, 'samautopick.py'). All particles were then displayed in SamViewer, in the order of their cross-correlation values from MRA, and a threshold was interactively set to remove the particles with cross correlation values below the threshold. A small number of particles with blurry appearance or with obvious wrong shape and size were also removed interactively We picked 70,585 particles from 300 cryo-EM images collected on the TF20, and 97,166 particles from 946 image stacks collected on the TF30. The selected particles were further screened by a reference-free $2 \mathrm{D}$ classification. A total of 45,625 particles from the TF20 data and 88,915 particles from the TF30 data were kept for determinations of $3 \mathrm{D}$ reconstructions.

All 3D classification and refinement were carried out in RELION ${ }^{52}$. The RCT $3 \mathrm{D}$ reconstruction from negatively stained TRPV1 was low-pass filtered to $60 \AA$, and used as the starting model for 3D classification of the TF20 data. After 25 iterations of 3D classification with 6 classes, particles in the classes that produced similar 3D reconstructions were combined for 3D auto-refinement. In this step, the $3 \mathrm{D}$ reconstructions from the $3 \mathrm{D}$ classification were low-pass filtered to $60 \AA$ and used as the starting models. 10,357 particles were used to calculate the final map (Extended Data Fig. 5). The resolution was estimated by the gold-standard FSC $=0.143$ criteria, after applying a soft spherical mask on the two reconstructions refined from the half-data sets independently ${ }^{23}$. The resolution of the $3 \mathrm{D}$ reconstruction from TF20 data was estimated as $8.8 \AA$. This $3 \mathrm{D}$ reconstruction was again low-passed filtered to $60 \AA$, and used as the starting model for 3D classification of the TF30 Polara data. 3D classification and refinement were carried out using the same procedure as for the TF20 data. The 3D reconstruction calculated from selected 35,645 particles each averaged from 30 subframes of motion-corrected image stack reached a resolution of 3.6 $\AA$. Continued refinement using the same particles but averaged from subframe 3-16 improved the resolution further to $3.4 \AA$. This was because the first 2 frames contain the most severe motion and the later frames accumulate more radiation damage ${ }^{15}$. The accumulated dose of the first 16 frames is $21 \mathrm{e}^{-} / \AA^{2}$ on specimen. Final gold-standard FSC curve was calculated 
using a soft spherical mask (with a 5-pixel fall-off) on the two independent reconstructions (Extended Data Fig. 8). The accuracies of rotation and translation reported by RELION were $3.54^{\circ}$ and $1.358 \AA$. RELION post-processing with auto-mask and auto-bfactor determined the resolution of the final map as $3.28 \AA$ and bfactor as $-101.228 \AA^{2}$. For model building and visualization, amplitude of the final 3D density map of $3.4 \AA$ resolution was amplified either by a temperature factor, $-100 \AA^{2}$ (Fig. 5) or by frequency-dependent scaling factor determined by comparing the experimental $3 \mathrm{D}$ density map with the ideal density map calculated from the atomic model (Figs 1d-f, 6a) ${ }^{15}$. All maps shown in the figures were low-pass filtered to the stated resolutions. The map deposited to the EMDB database is the raw map without amplitude sharpening, masking or filtering. UCSF Chimera ${ }^{53}$ was used to visualize and segment the cryo-EM maps, and the rigid body fitting of atomic model was done using the fit-in-map function from Chimera.

Model building in Coot. At $3.4 \AA$ resolution, the cryo-EM map was of sufficient quality for de novo atomic model building, except for the ARD, where the density is not as well resolved as other regions of the channel. Taking advantage of the defined geometry of $\alpha$-helices and clear bumps for $\mathrm{C} \alpha$ atoms, a polyalanine model was first built in $\operatorname{Coot}^{54,55}$. Amino acid assignment was subsequently achieved based mainly on the clearly defined side chains densities of bulky residues such as Phe, Tyr and Trp, as well as some Arg and Lys residues. The ARD structure (PDB 2PNN) was docked into the density as a rigid body without further modification. The final model includes almost all residues, except for those located at S2-S3 loop (503-507) and a region C-terminal to the TRP domain (720-751). Although a C-terminal region of the channel (registered as 752-762 in the model) clearly assumes a $\beta$-strand secondary structure, amino acids could not be assigned since side chain density is not well resolved at this segment. The final model exhibits good geometry as indicated by the Ramachandran plot (preferred region, $95.73 \%$; allowed region, $3.92 \%$; outliers, $0.34 \%$ ). Of note, the six transmembrane helices, TRP domain and pre-S1 helix are devoid of any such outliers. One outlier is located at the S3-S4 loop and the other resides at docked ankyrin repeats. Pore radii were calculated using the HOLE program ${ }^{56}$.

45. Dukkipati, A., Park, H. H., Waghray, D., Fischer, S. \& Garcia, K. C. BacMam system for high-level expression of recombinant soluble and membrane glycoproteins for structural studies. Protein Expr. Purif. 62, 160-170 (2008).

46. Reeves, P. J., Callewaert, N., Contreras, R. \& Khorana, H. G. Structure and function in rhodopsin: high-level expression of rhodopsin with restricted and homogeneous $\mathrm{N}$-glycosylation by a tetracycline-inducible $\mathrm{N}$-acetylglucosaminyltransferase I-negative HEK293S stable mammalian cell line. Proc. Natl Acad. Sci. USA 99, 13419-13424 (2002).

47. Althoff, T., Mills, D. J., Popot, J. L. \& Kuhlbrandt, W. Arrangement of electron transport chain components in bovine mitochondrial supercomplex $\left.\right|_{1}\left|I_{2}\right| V_{1}$. EMBO J. 30, 4652-4664 (2011).

48. Popot, J. L. et al. Amphipols from A to Z. Annu. Rev. Biophys. 40, 379-408 (2011).

49. Mindell, J. A. \& Grigorieff, N. Accurate determination of local defocus and specimen tilt in electron microscopy. J. Struct. Biol. 142, 334-347 (2003).

50. Frank, J. et al. SPIDER and WEB: processing and visualization of images in 3D electron microscopy and related fields. J. Struct. Biol. 116, 190-199 (1996).

51. Grigorieff, N. FREALIGN: high-resolution refinement of single particle structures. J. Struct. Biol. 157, 117-125 (2007).

52. Scheres, S. H. RELION: implementation of a Bayesian approach to cryo-EM structure determination. J. Struct. Biol. 180, 519-530 (2012).

53. Pettersen, E. F. etal.UCSF Chimera-a visualization system for exploratory research and analysis. J. Comput. Chem. 25, 1605-1612 (2004).

54. Emsley, P. \& Cowtan, K. Coot: model-building tools for molecular graphics. Acta Crystallogr. D 60, 2126-2132 (2004)

55. Emsley, P., Lohkamp, B., Scott, W. G. \& Cowtan, K. Features and development of Coot. Acta Crystallogr. D 66, 486-501 (2010)

56. Smart, O. S., Neduvelil, J. G., Wang, X., Wallace, B. A. \& Sansom, M. S. HOLE: a program for the analysis of the pore dimensions of ion channel structural models. J. Mol. Graph. 14, 354-360 (1996). 

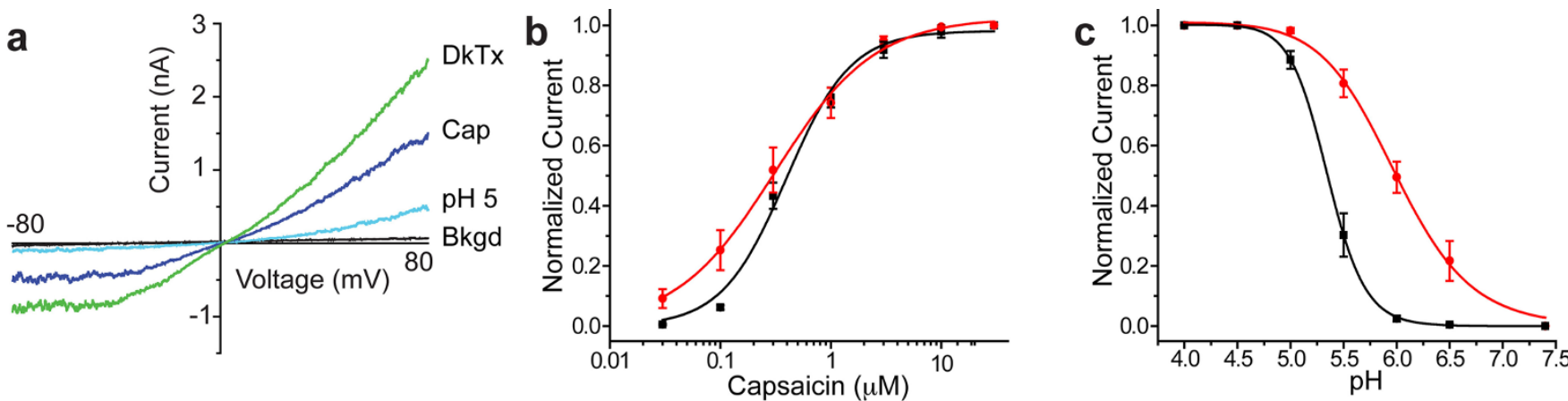

f

\begin{tabular}{|l|c|c|c|}
\hline & Capsaicin & DkTX & RTX/DkTX \\
\hline $\mathrm{P}_{\mathrm{C2} 2+} / \mathrm{P}_{\mathrm{Na}+}$ & 4.8 & 5.6 & 5.5 \\
\hline $\mathrm{P}_{\mathrm{K}+} / \mathrm{P}_{\mathrm{Na}+}$ & 0.96 & N.D. & N.D. \\
\hline $\mathrm{P}_{\mathrm{Cs}+} / \mathrm{P}_{\mathrm{Na}+}$ & 0.91 & N.D. & N.D. \\
\hline
\end{tabular}
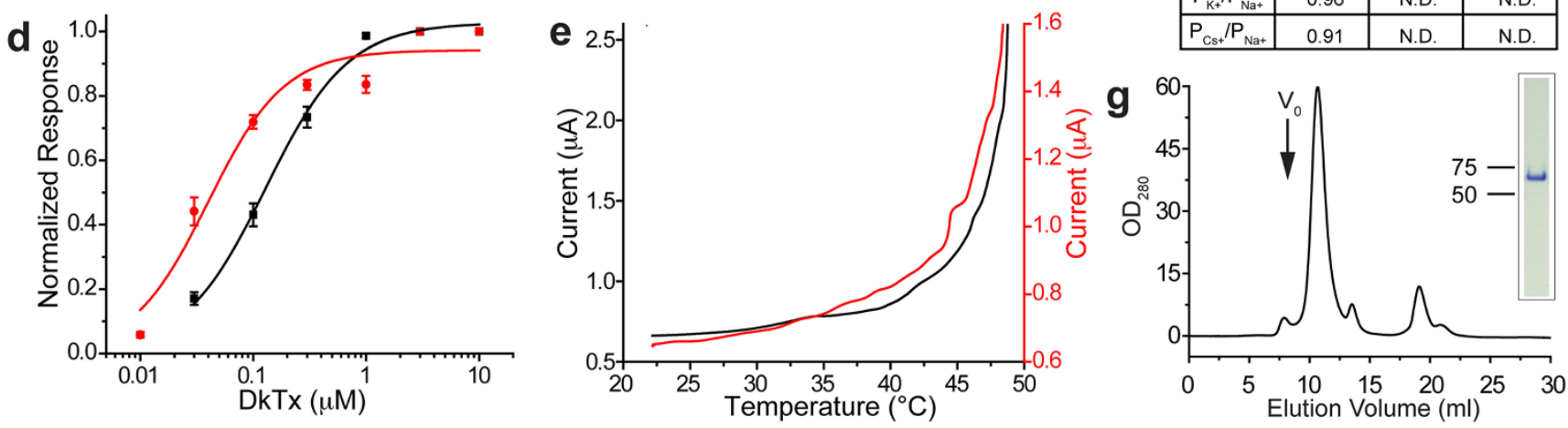

Extended Data Figure $1 \mid$ A minimal TRPV1 channel that is functional and biochemically stable. a, Mammalian (HEK293) cells expressing a minimal construct (with an $\mathrm{N}$-terminal green fluorescent protein (GFP) tag) responded to various TRPV1 agonists, including capsaicin (Cap; $0.5 \mu \mathrm{M}$ ), extracellular protons ( $\mathrm{pH} 5.0$ ) and double-knot spider toxin (DkTx; $2 \mu \mathrm{M}$ ). Electrophysiological responses were measured in whole-cell patch-clamp configuration. b, c, Dose-responsive curves for capsaicin (b) or protons (c) were determined for minimal (black) or full-length (red) TRPV1, both of which contained an N-terminal GFP fusion. Values were normalized to maximal currents evoked by $30 \mu \mathrm{M}$ capsaicin (b) or $\mathrm{pH} 4.0$ (c) $(n=6$ independent whole-cell recordings). d, DkTx dose-response curves for minimal (black) or full-length (red) TRPV1 as in $\mathbf{b}$ and $\mathbf{c}$, determined by calcium imaging. Values were normalized to maximal capsaicin

$(10 \mu \mathrm{M})$-evoked response in transfected HEK293 cells $(n>30$ per point). e, Thermal response profiles for minimal (black) or full-length (red) TRPV1-expressing oocytes reveal similar heat sensitivity. f, Ion permeability ratios of agonist-evoked currents from minimal TRPV1 were estimated from reversal potential shifts in whole-cell patch-clamp recordings of transfected HEK293 cells, revealing no significant differences from full-length channel. g, Gel-filtration profile (Superdex-200) of detergent solubilized TRPV1 after purification on amylose affinity resin and proteolytic removal of maltose-binding protein (MBP) tag. The major species elutes as a symmetrical peak after the void volume $\left(\mathrm{V}_{0}\right)$. Inset shows that peak material migrates as a single, homogeneous band on SDS-PAGE (4-12\% gradient gel; Coomassie stain). 


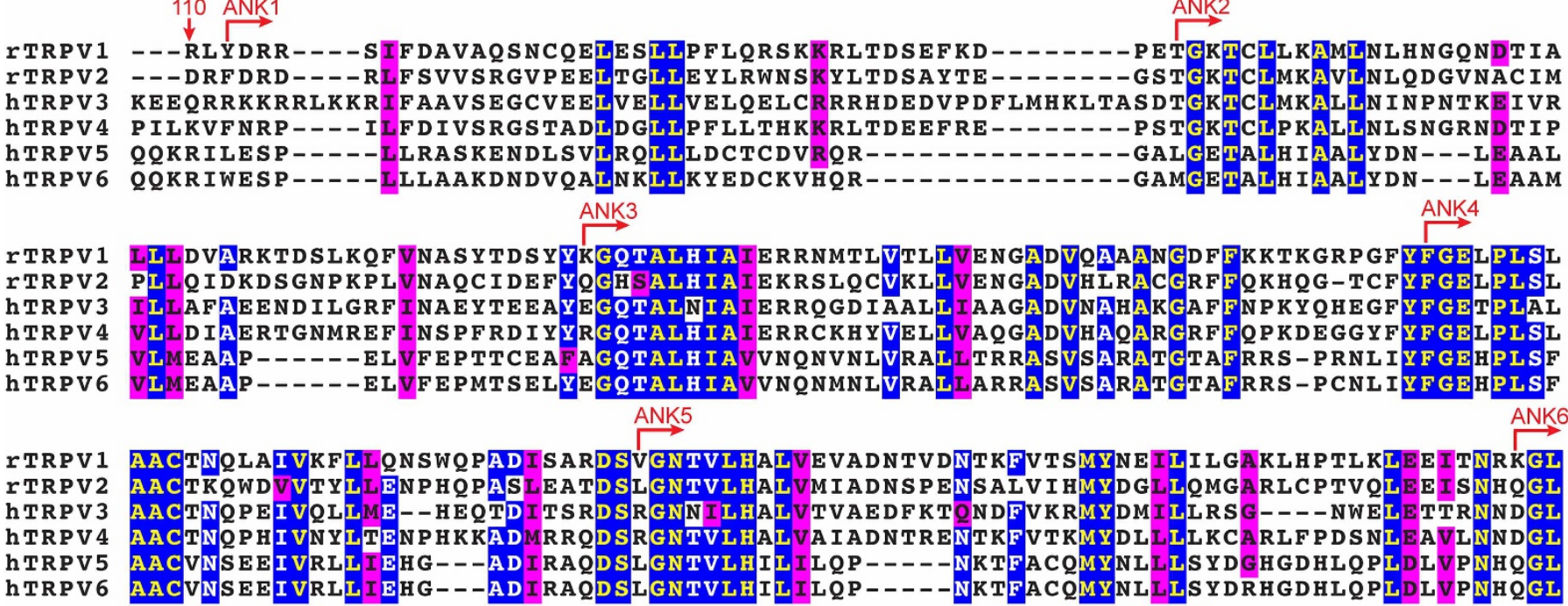

TTRPV 1 TPLALAAS SGK I GVLAYILQRE I HEPECRHLSRKF TEWAYGPVHSSLYDLSCIDTC-EKNSVLEVIAYSSSETPNRHDML ITRPV2 TPLKLAAKEGKIEIFRHILQREFSGP-YQPLSRKFTEWCYGPVRVSLYDLSSVDSW-EKNSVLEIIAFHCK-SPNRHRMV hTRPV3 TPLQLAAKMGKAEILKYILSREIKEKRLRSLSRKFTDWAYGPVSSSLYDLTNVDTT-TDNSVLEITVYNTN-IDNRHEML hTRPV4 SPLMMAAKTKIGIFQHIIRREVTDEDTRHLSRKFKDWAYGPVYSSLYDLSSLDTCGEEASVLEILVYNSK-IENRHEML hTRPV5 TPFKLAGVEGNTVMFQHLMQ--_..--KRRH I QWTYGPLTSILYDLTEIDSWGEELSFLELVVSSDK--REARQIL hTRPV6 TPFKLAGVEGNTVMFQHLMQ---.----KRKHTQWTYGPLTSTLYDLTEIDSSGDEQSLLELI ITTKK--REARQIL Pre-S1Helix

ITRPV 1 LVEPLNRLLQDKWDRFVKRIFYFNFFVYCLYMIIFTAAAYYRPVEG-_- _ _ _ _ _ _ - LPY KLKNTVGDYFRVTG

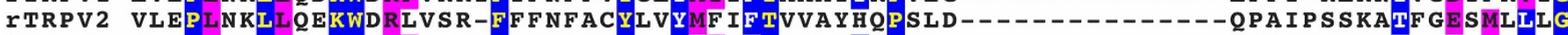

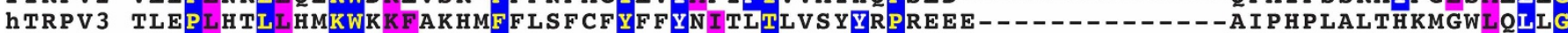
hTRPV4 AVEPINELLRDKWRKFGAVSFY INVVSYLCAMVIFTLTAYYQPLEG-- - - - - - - - - TPPYP- - YRTTVDYLRLAG hTRPV5 EQTPVKELVSFKWNKYGRPYFCILAALYLLYMICFTTCCVYRPLKFRGGNRTHSRDITILQQKLLQEAYETREDIIRLVG hTRPV 6 DQTPVKELVSLKWKRYGRPYFCMLGAIYLLYI ICFTMCCIYRPLKPRTNNRTSPRDNTLLQQKLLQEAYMTPKDDIRLVG S2

rTRPV1 EILSVSGGVYFFFRGIQ-YFLO-RRPSLKSLFVDSYSEILFFVOSLFMLVSVVLYF S ORKEYVAS MVF SLAMGWTNMLYY rTRPV2 HILILLGGIYLLLGQLW-YFWR-RRLFIWISFMDSYFEILFLLQALLTVLSQVLRFMETEWYLPLLVLSLVLGWLN hTRPV3 RMFVLIWAMCISVKEGIAIFLL-RPSDLQSILSDAWFHFVFFIQAVLVILSVFLYLAYKEYLACLVLAMALGWAN hTRPV4 EVITLFTGVLFFFTNTKDLFMK-KCPGVNSLFIDGSFQLLYFIYSVLVIVSAALYLAGIEAYLAVMVFALVLGWMNAI hTRPV5 ELVSIVGAVIILLLEIPDIFRVGASRYFGKTILGGPFHVIIITYASLVLVTMVMRLTNTNGEVVPMSFALVLGWCSVMY hTRPV6 ELVTVIGAIILLVEVPDIFRMGVTRFFGTILGGPFHVLIITYAFM S4-S5 Linker S5

ITRPV 1 TRGFOOMGI YAVMIEKMILRDLCLFMFVYLVFLFGFSTAVVTLIEDGKNNSLPMES----TPHKCRGSACKPGNSYNSLY YTRPV2 TRGFQHTGI Y SVIQKVILRDLLRFLLVYLVFLFGFAVALVSLSREARSPKAPEDNNSTVTEQPTVGQEEEP-APYRS IL hTRPV 3 TRGFOSMGMY SVMIOKVILHDVLKFLFVY IVFLLGFGVALASLIEKCPK-_hTRPV4 TRGLKLTGTYSIMIQKILFKDLFRFLLVYLLFMIGYASALVSLLNPCANMKVCNED----OTNCTVPTYPSCRDSETFS hTRPV5 TRGFQMLGPFTIMIQKMIFGDLMRFCWLMAVVILGFASAFYI IFQTE--_-_. hTRPV6 ARGFOMLGPFTIMIQKMIF GDLMRFCWLMAVVILGFASAFYI IFQTE-_Pore Helix Y565 Selectivity Filter

RTRPV1 STCLELFKF TIGMGDLE F TENY DF KAVF I ILLLAYVILTYILLLNMLIALMGETVNKIAOES KNIWKLORAITILDTEKS YTRPV2 DASLELFKFTIGMGELAFQEQLRFRGVVLLLLLAYVLLTYVLLLNMLIALMSETVNHVADNSWS I WKL QKAISVLEMENG hTRPV3 DAVLELFKLTIGLGDLNIQQNSKYPILFLFLLITYVILTFVLLLNMLIALMGETVENVSKESERIWRLORARTILEFEKM hTRPV 4 TFLLDLFKLTIGMGDLEMLSSTKYPVVFIILLVTYIILTFVLLLNMLIALMGETVGOVSKESKHIWKLOWATTILDIERS hTRPV5 MALFTTFELFLTVIDAPANYDVDLPFMF I VNFAFAIIATLLMLNLFIAMMGDTHWRVAQERDELWRAOVVATTVMLERK hTRPV 6 MALFSTFELFLTI IDGPANYNVDLPFMYSITYAAFAI IATLLMLNLLIAMMGDTHWRVAHERDELWRAOIVATTVMLERK $\underline{\mathrm{G} 643}$

$$
\underline{1679} \quad 764 \quad \underline{W 697}
$$

$\downarrow$

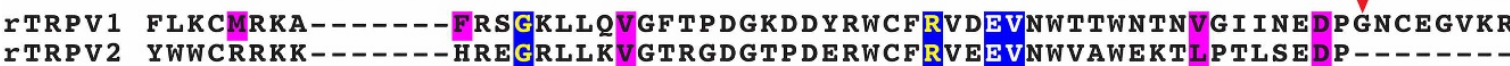
hTRPV3 LPEWLRSR------FRMGELCKVA----EDDFRLCLRINEVKWTEWKTHVSFLNEDP-hTRPV 4 FPVFLRKA------ FRSGEMVTVGKSSDGTPDRRWCFRVDEVNWSHWNQNLGIINEDPGKNE-TYQ hTRPV5 LPRCLWPRSGICGCEFGLGDRWFLRVENHNDQNPLRVLRYVEVFKNSDKEDDQEHPSEKQPSG--- hTRPV6 LPRCLWPRSGICGREYGLGDRWFLRVEDRQDLNRQRIQRYAQAFHTRGSEDLDKDSVEK-_- -

Extended Data Figure $2 \mid$ Sequence alignment of TRPV1 to other TRPV family members. The rat TRPV1 construct used for this study consists of residues 110 to 764 (indicated by red arrows), excluding the highly divergent region (604-626, highlighted by cyan box). Secondary structure elements are indicated above the sequence. The starting points of six ankyrin repeats are based on a crystal structure of ARD of TRPV1 (PDB 2PNN). Several critical residues discussed in the text are labelled in blue, and conserved glycine and proline residues at the turn of a $\beta$-sheet (highlighted in Fig. 6) are indicated with red stars. 

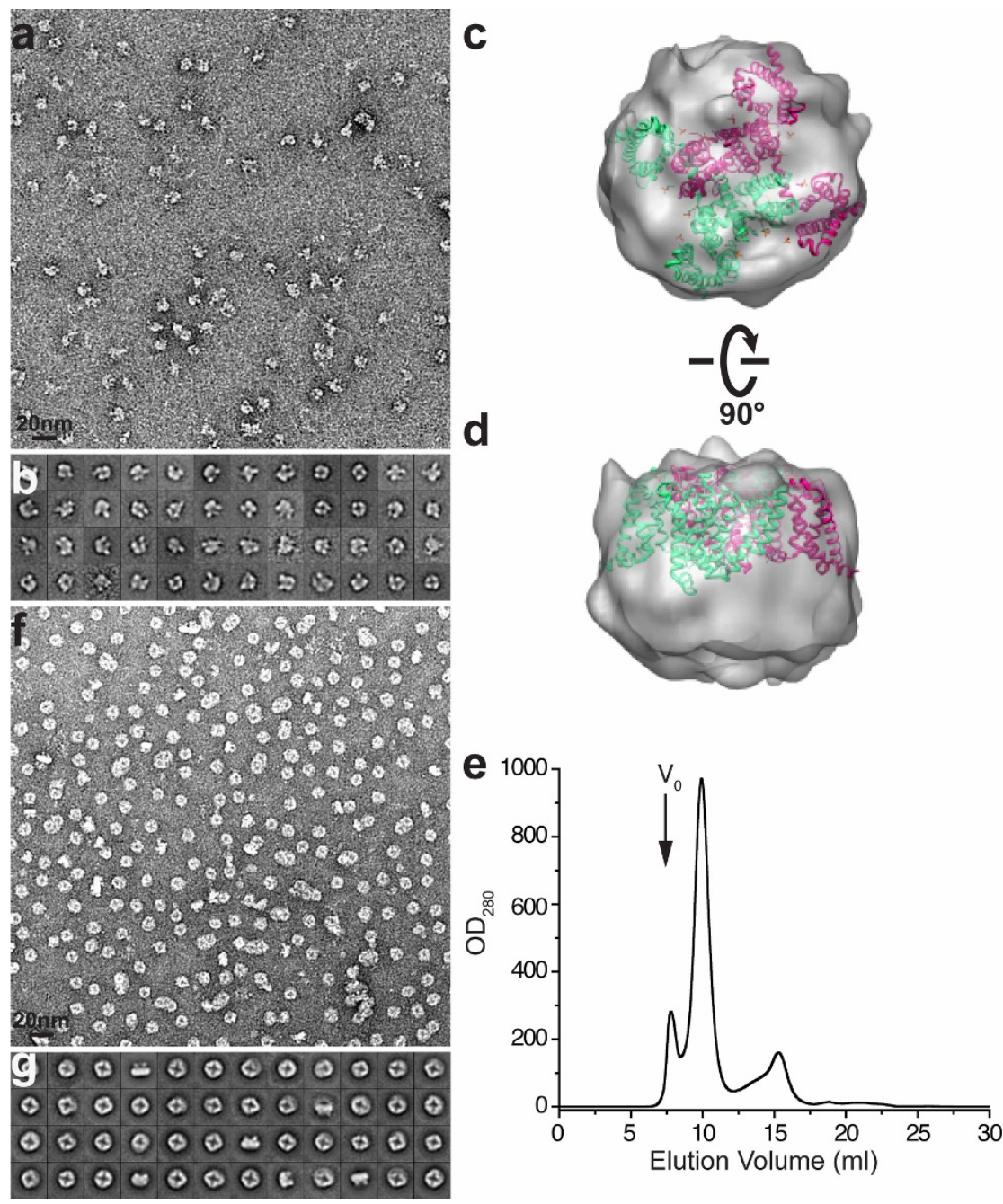

Extended Data Figure $3 \mid$ Negative-stain EM of TRPV1. a, Representative negative-stain image of purified minimal TRPV1 protein in detergent (n-dodecyl $\beta$-d-maltopyranoside; DDM) after proteolytic removal of MBP tag. b, 2D class averages of negatively stained particles in DDM. c, d, Two views of a random conical tilt (RCT) reconstruction from negatively stained TRPV1 in DDM. The RCT reconstruction was low-pass filtered at $30 \AA$, and fitted with

the structure of $\mathrm{Na}_{V} \mathrm{Ab}$ (PDB 3RVY) to indicate the size and general shape e, Gel-filtration profile (Superdex-200) of purified minimal TRPV1 protein after exchange from DDM into amphipols. The major species elutes as a symmetrical peak after the void volume $\left(\mathrm{V}_{0}\right)$. f, Representative negative-stain image of purified minimal TRPV1 protein without MBP tag in amphipols. g, 2D class averages of negative-stain particles in amphipols. 


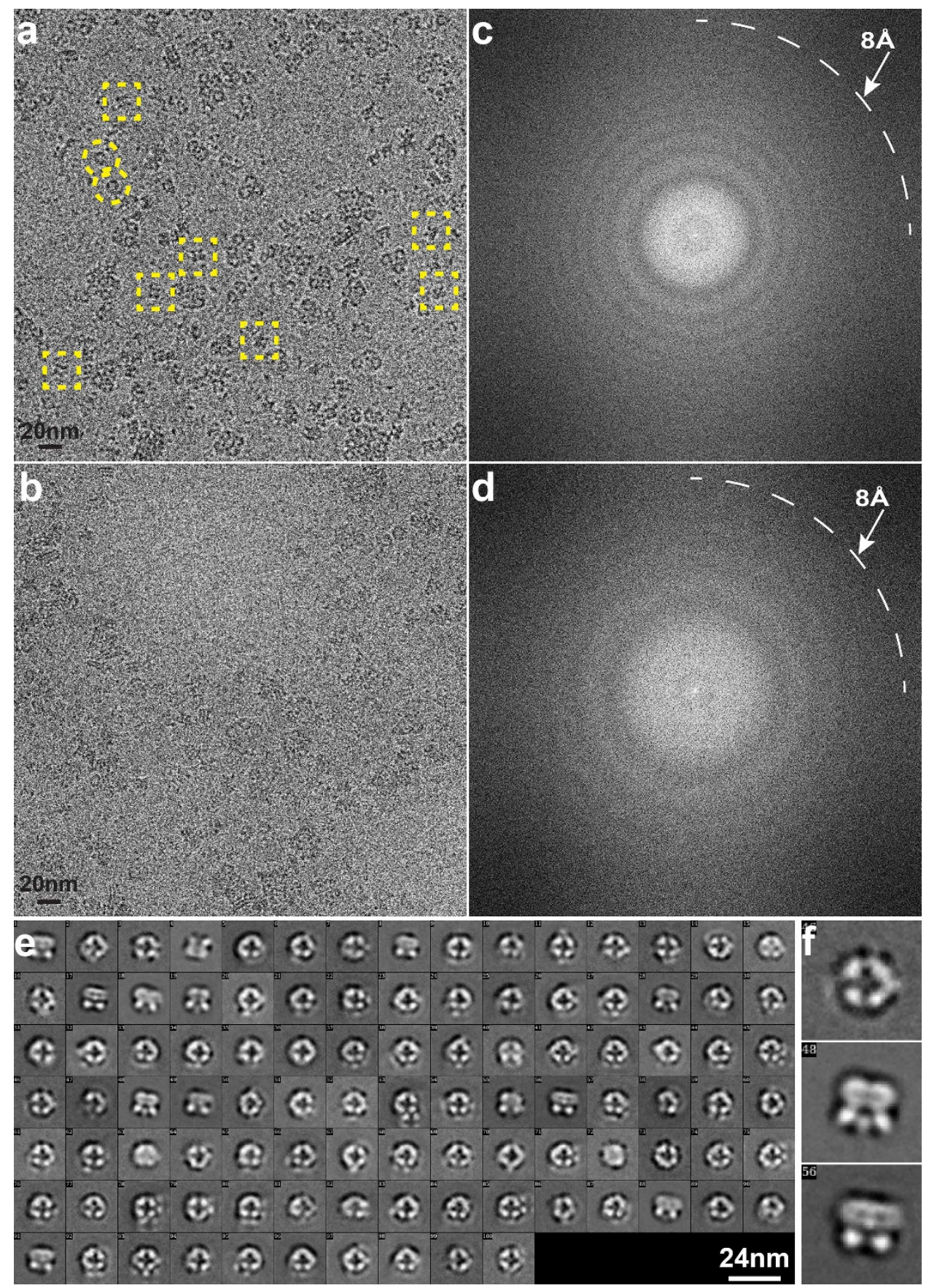

Extended Data Figure $4 \mid$ Cryo-EM of TRPV1 using Tecnai TF20 microscope and TemF816 8k $\times 8 \mathrm{k}$ CMOS camera. a-d, Representative images of frozen hydrated TRVP1 in amphipols taken at different defocus levels, $3.1 \mu \mathrm{m}$ (a) and $1.5 \mu \mathrm{m}$ (b) and their Fourier transforms (c, d). Thon rings extend to $\sim 8 \AA$. Dash-line squares or circles indicate representative particles showing two distinctive views. e, 2D class averages of TRPV1 particles. f, Enlarged view of three representative $2 \mathrm{D}$ class averages. 


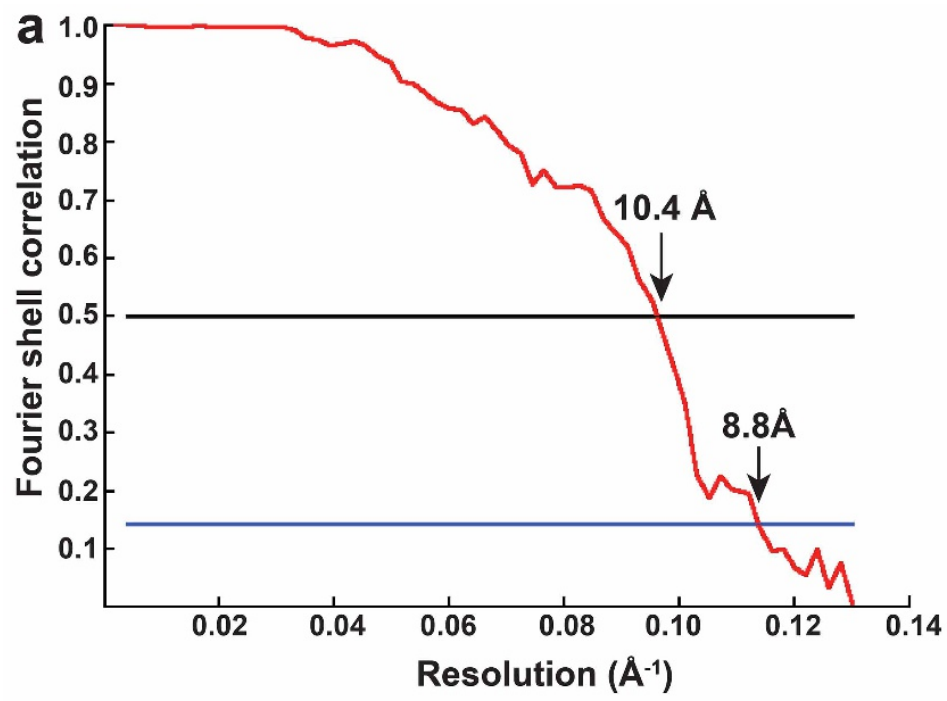

b
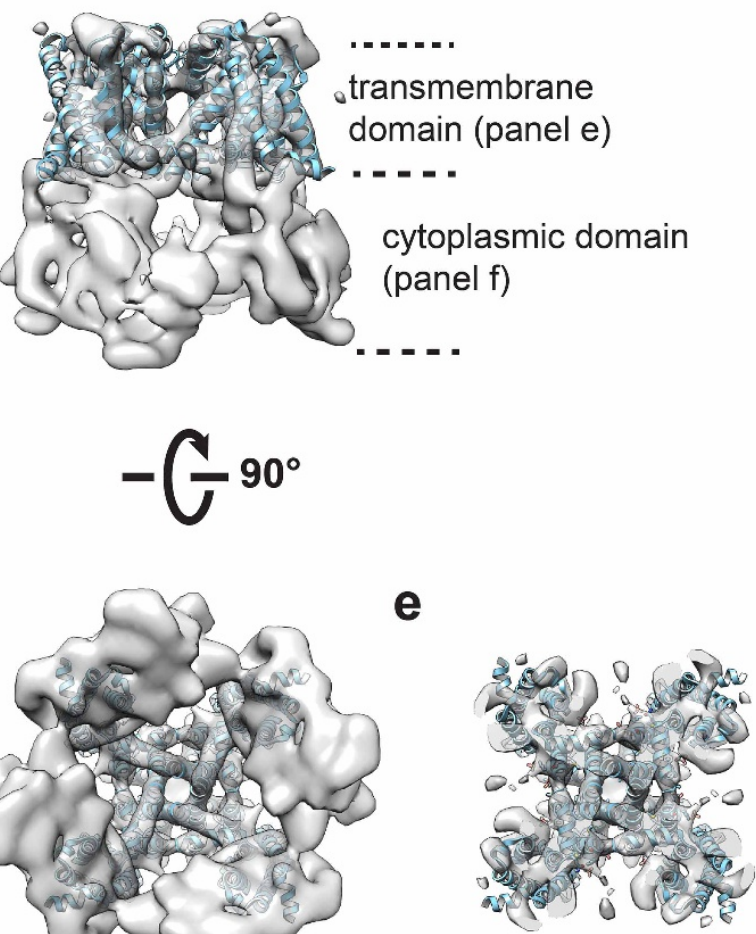

C

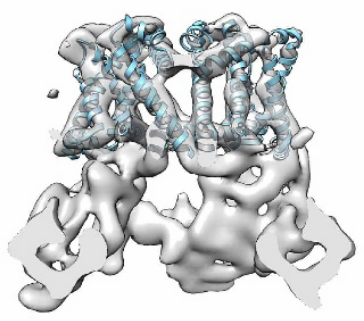

$-\left(\frac{1}{9} 90^{\circ}\right.$

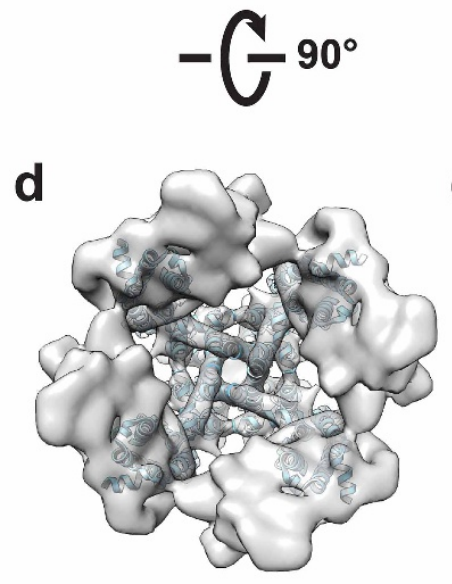

e

Extended Data Figure $5 \mid$ 3D reconstruction of TRPV1 calculated from TF20 data. a, Gold-standard FSC curve for the 3D reconstruction, marked with resolutions corresponding to FSC $=0.5$ and 0.143 . b. Side view of the $3 \mathrm{D}$ reconstruction low-pass filtered at $9 \AA$ and amplified by a temperature factor $-1,500 \AA^{2}$, showing transmembrane (top) and cytoplasmic (bottom) domains.
The transmembrane domain roughly fitted by the atomic model of $\mathrm{Na}_{V} \mathrm{Ab}$ (PDB 3RVY). c, Longitudinal cross section view focused on central transmembrane helices. d, Bottom-up view of the $3 \mathrm{D}$ reconstruction shows overall structure. e, f, Bottom-up cross-section views showing the arrangement of transmembrane (e) and cytoplasmic (f) domains. 

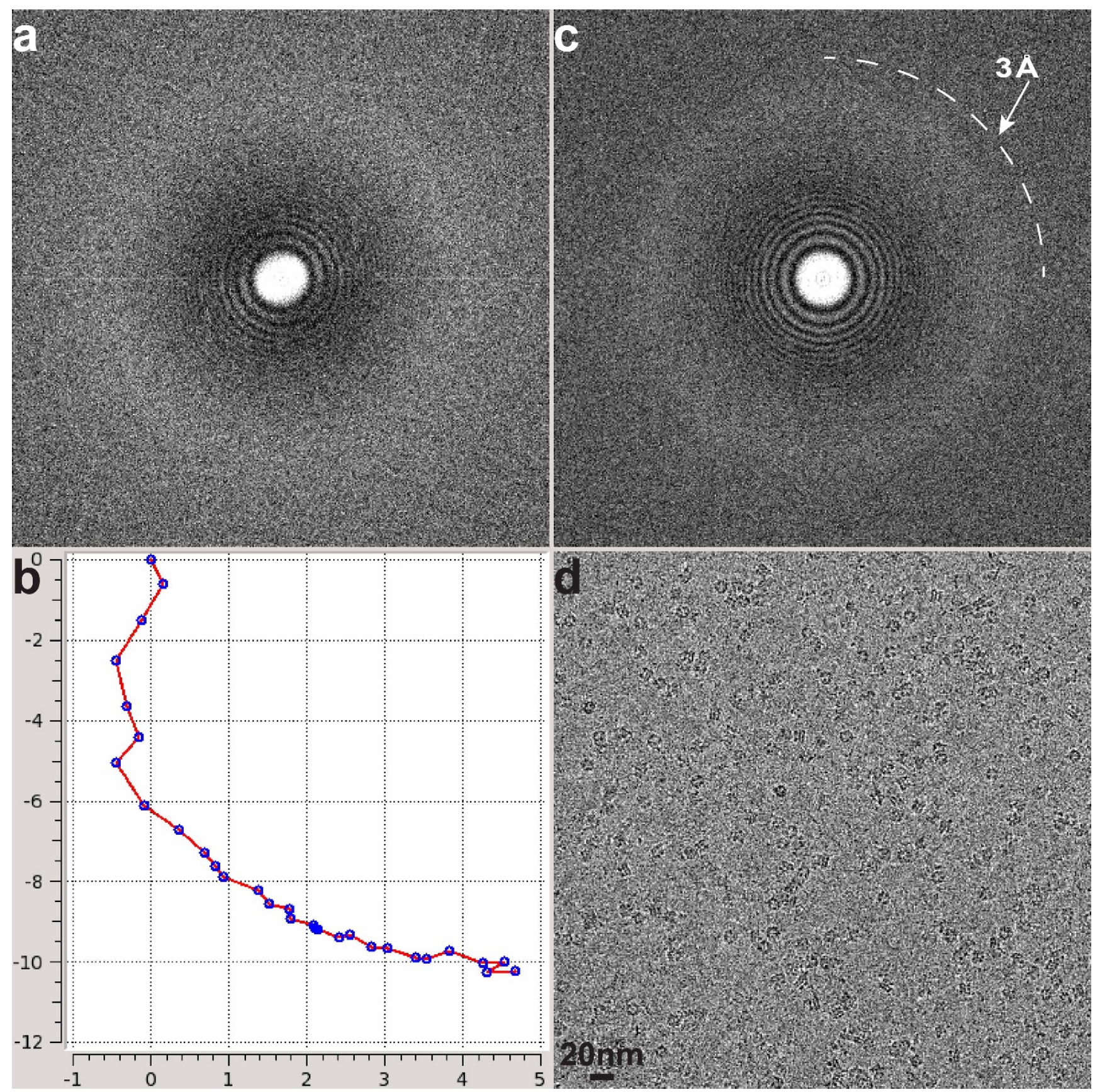

Extended Data Figure 6 Motion correction improves the quality of images collected on Polora TF30 microscope using a K2 Summit direct electron detector. a, Fourier transform of a representative cryo-EM image of TRPV1 embedded in a thin layer of vitreous ice over Quantifoil hole without supporting

carbon film before motion correction. $\mathbf{b}$, Path of motion of 30 individual subframes, determined as described in Methods. c, d, A nearly perfect Fourier transform (c) was restored after the EM image was corrected for motion (d). 


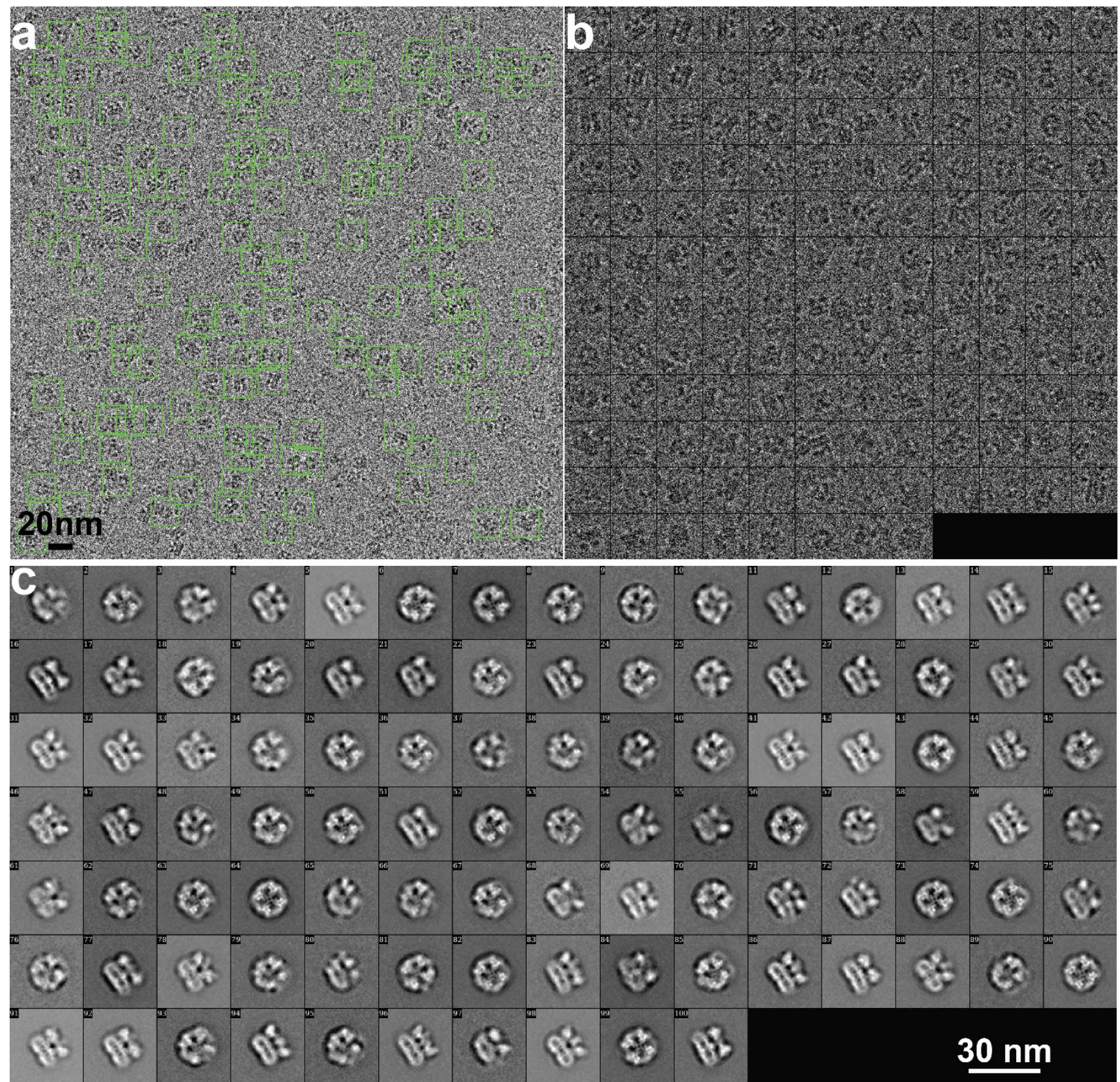

Extended Data Figure $7 \mid$ Picking and 2D classification of TRPV1 Cryo-EM particles collected on Polora TF30 microscope. a, Representative cryo-EM image after motion correction. Green boxes indicate all particles that were selected by semi-automatic particle picking and 2D screening, as described in
Methods. b, Gallery view of the particles shown in a. c, 2D class averages of cryo-EM particles show many fine features (also seen in enlarged views in Fig. 1c), and these features are not visible in the 2D class averages of cryo-EM particles from TF20 data (Extended Data Fig. 4e, f). 

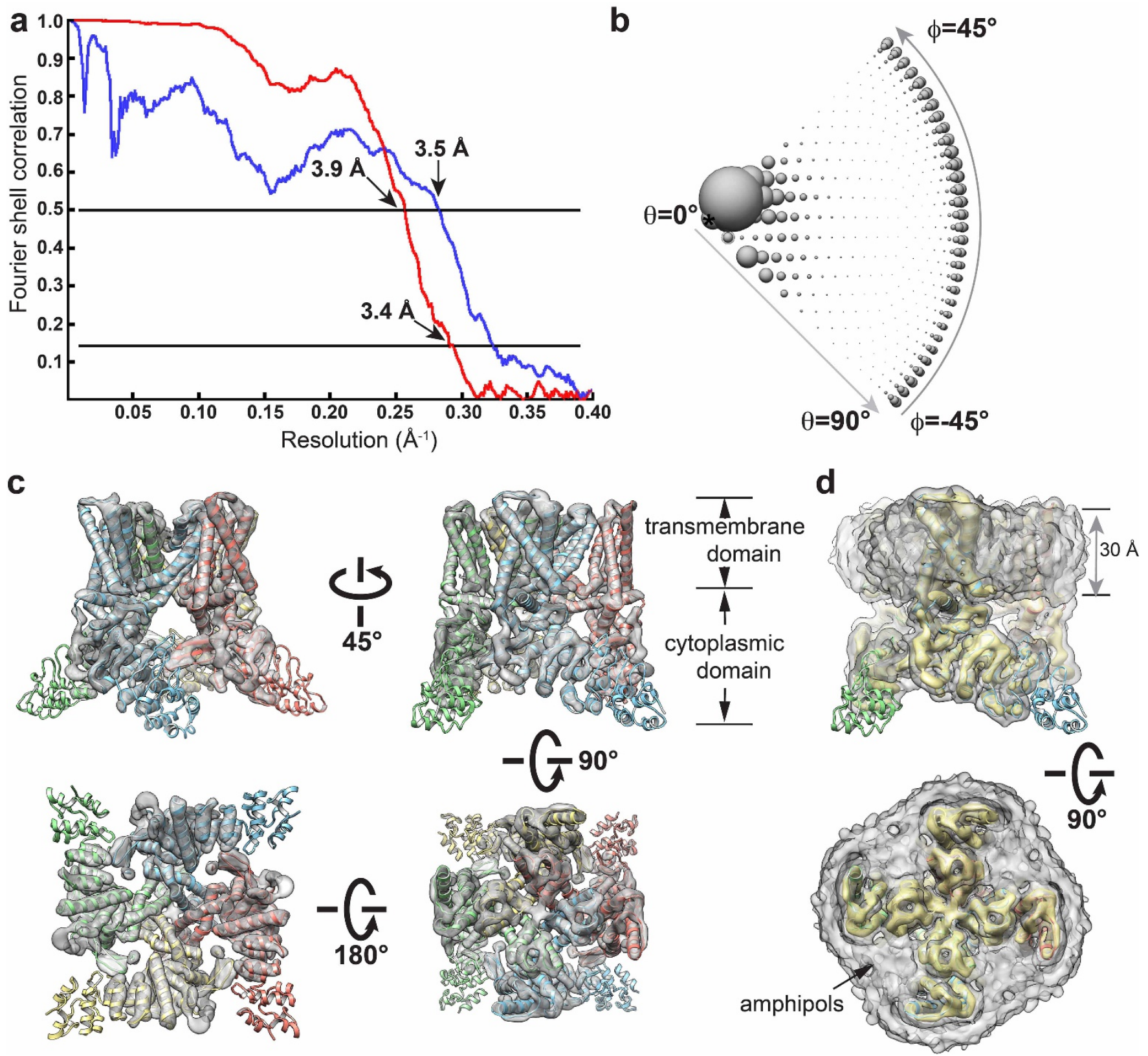

Extended Data Figure $8 \mid 3$ D reconstruction of TRPV1 calculated from TF30 data. a, Gold-standard FSC curve (red) of the final 3D reconstruction, marked with resolutions corresponding to FSC $=0.5$ and 0.143 . The FSC curve between the final map and that calculated from the atomic model is shown in blue. The relative low value of this FSC (blue) at low frequency range $(>10 \AA)$ is probably due to the presence of amphipol density in the experimental map. b, Euler angle distribution of all particles used for calculating the final $3 \mathrm{D}$ reconstruction. The sizes of balls represent the number of particles. The accuracy of rotation is $3.54^{\circ}$, as reported by RELION. c, Different views of the 3D reconstruction low-pass filtered at $6 \AA$ and amplified by a temperature factor of $-100 \AA^{2}$, fitted with the atomic model of TRPV1. d, Two views of the $3 \mathrm{D}$ reconstruction displayed at two different isosurface levels (high in yellow and low in grey). At the low isosurface level, the belt-shaped density of amphipols is visible with a thickness of $\sim 30 \AA$. 
a

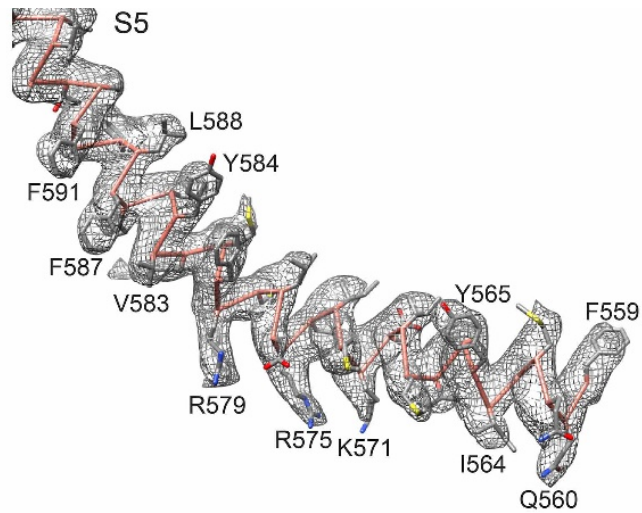

S4-S5 linker

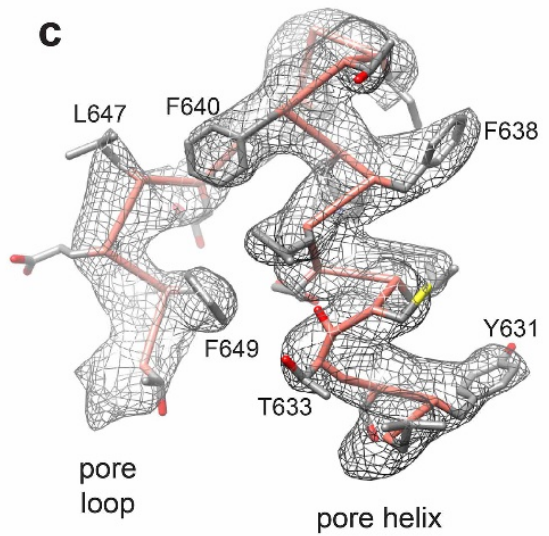

e

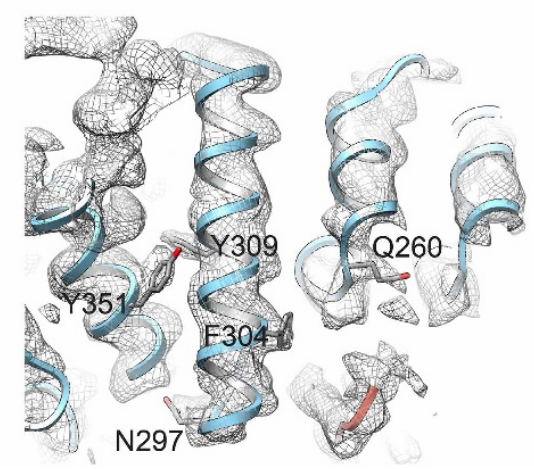

Extended Data Figure $9 \mid$ Cryo-EM densities of selected regions of TRPV1 at 3.4 $\mathrm{A}$ resolution. a-d, Representative cryo-EM densities (grey mesh) are superimposed on atomic model (main chain in pink) for various TRPV1 domains, as indicated. e, f, Representative cryo-EM densities (grey mesh) are
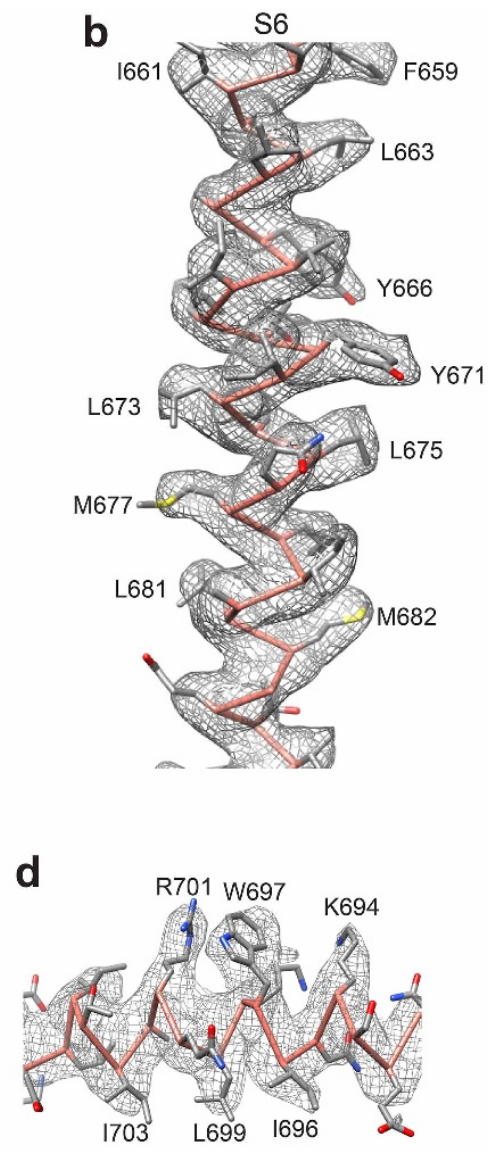

TRP domain docked with crystal structure of TRPV1 ankyrin repeats (PDB 2PNN).

Accuracy of docking was supported by fitting of several bulky side chains. Map was low-pass filtered to $3.4 \AA$ and amplified by a temperature factor $-100 \AA^{2}$. 

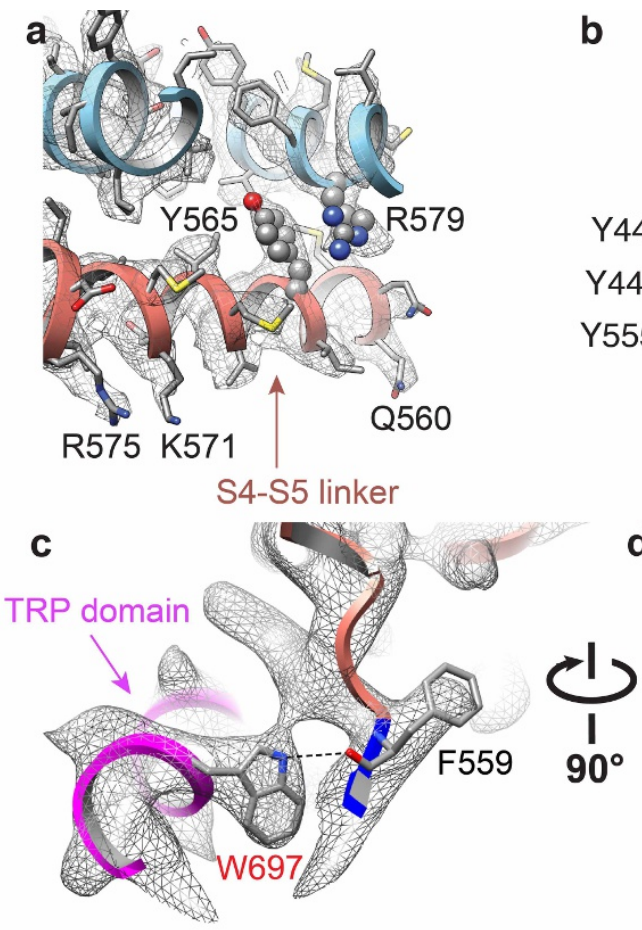

e

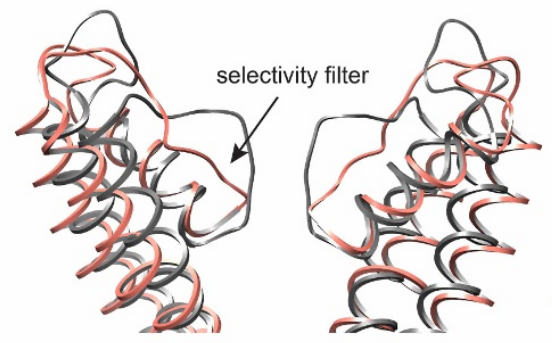

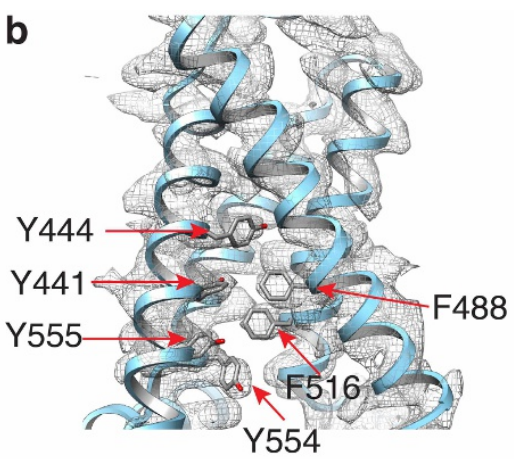

d

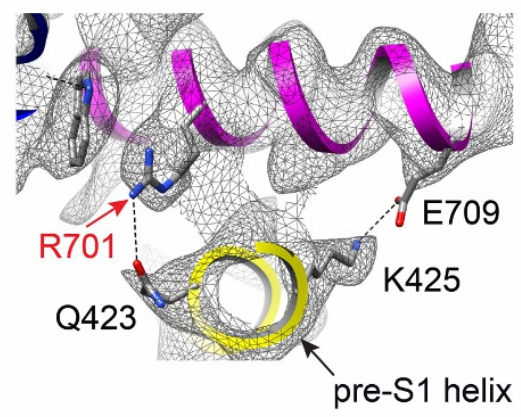

f

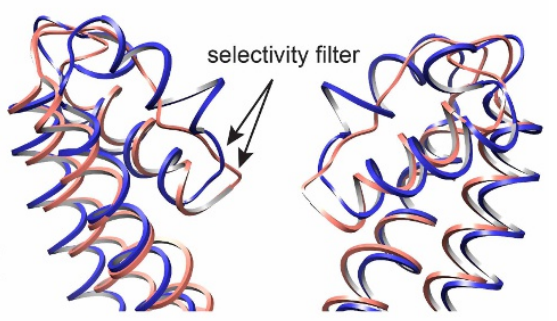
$\mathrm{Na}_{\mathrm{A}} \mathrm{Ab}$

Extended Data Figure $10 \mid$ Details of domain interactions and outer pore configurations. a-d, Cryo-EM densities (grey mesh) of highlighted regions of TRPV1, as indicated, at $3.4 \AA$ resolution are superimposed onto atomic model. Map was low-pass filtered to $3.4 \AA$ and amplified by a temperature factor $-100 \AA^{2}$. e, Superimposition of TRPV1 (salmon) with $K_{V} 1.2-2.1$ chimaera
(PDB 2R9R; grey). f, Superimposition of TRPV1 (salmon) with $\mathrm{Na}_{\mathrm{V}} \mathrm{Ab}$ (PDB 3RVY; blue). In each case, substantial structural differences are observed in the outer pore region. Structural alignments are based on the pore domain (S5-P-S6). 